\title{
Lifetime consumption-portfolio choice under trading constraints, recursive preferences, and nontradeable income
}

\author{
Mark Schroder $^{\mathrm{a}}$, Costis Skiadas ${ }^{\mathrm{b}, *}$ \\ ${ }^{a}$ Department of Finance, Eli Broad Graduate School of Management, Michigan State University, \\ 323 Eppley Center, East Lansing, MI 48824, USA \\ ${ }^{\mathrm{b}}$ Department of Finance, Kellogg School of Management, Northwestern University, \\ 2001 Sheridan Road, Evanston, IL 60208, USA
}

Received 21 April 2004; received in revised form 19 July 2004; accepted 5 August 2004 Available online 11 September 2004

\begin{abstract}
We analyze the lifetime consumption-portfolio problem in a competitive securities market with continuous price dynamics, possibly nontradeable income, and convex trading constraints. We define a class of "translation-invariant" recursive preferences, which includes additive exponential utility, but also nonadditive recursive and multiple-prior formulations, and allows for first and second-order source-dependent risk aversion. For this class, we show that the solution reduces to a single constrained backward stochastic differential equation, which for an interesting class of incomplete-market problems simplifies to a system of ordinary differential equations of the Riccati type.

(C) 2004 Elsevier B.V. All rights reserved.
\end{abstract}

Keywords: Finance; Optimal portfolios; Recursive utility; BSDE; FBSDE

\footnotetext{
${ }^{*}$ Corresponding author. Tel.: + 1-847-467-2328; fax: + 1-847-491-5719.

E-mail addresses: schroder@bus.msu.edu (M. Schroder), c-skiadas@kellogg.northwestern.edu (C. Skiadas).
} 


\section{Introduction}

In this paper, we analyze the lifetime consumption-portfolio problem for an agent with a possibly nontradeable income stream who can trade in a competitive securities market with essentially arbitrary continuous price dynamics, under the constraint that the vector of risky-asset position values must lie in a given convex set at all times. Starting with the general first-order conditions of optimality, we consider increasingly restrictive and increasingly tractable formulations.

It is well known in economics that an easily solvable class of problems with nontradeable income arises with additive exponential utility and Gaussian dynamics (see, for example, [38]). Additivity of utility, however, is known to impose an ad hoc relationship between intertemporal substitution and risk aversion (see, for example, [12]). In particular, the risk aversion coefficient with expected discounted exponential utility is entirely determined by an agent's preferences over deterministic consumption plans.

A main contribution of this paper is to define translation-invariant recursive utility, a generalization of additive exponential utility that retains the tractability advantages of the latter, but frees the specification of absolute risk aversion from preferences over deterministic plans. Moreover, risk aversion can be different for every source of risk, for example, reflecting the source's ambiguity in the sense of Ellsberg [11], and also risk aversion can be either first or second order, in a dynamic version of the distinction made by Segal and Spivak [34]. For a special class of translation-invariant recursive utilities and dynamic investment opportunity sets, the Gaussian case included, the incomplete market problem reduces to a system of Riccati-type ordinary differential equations. The result is a modeling framework that, relative to the exponential-Gaussian benchmark, is significantly more flexible, yet still highly tractable.

This paper's analysis is a natural continuation of that in Schroder and Skiadas [32], hereafter abbreviated to SS. In the latter there is no endowed income stream (or if there is one, it is traded), portfolio constraints are imposed in terms of proportions of wealth, and the focus is on simplifications achieved through the assumption of scale invariance (or homotheticity), which is inconsistent with a nontradeable income stream. In contrast, in this paper constraints are imposed on dollar amounts, a nontradeable income is allowed, and the focus is on simplifications achieved by translation invariance (or quasilinearity) relative to a fixed plan. In SS we defined source-dependent relative risk aversion, while here we define source-dependent absolute risk aversion.

The first-order conditions of optimality with recursive utility take the form of a system of forward-backward stochastic differential equations (FBSDE), corresponding to a PDE system in a Markovian setting. The forward and backward components of the FBSDE system are shown to uncouple under a scale-invariance assumption in SS, and under a translation-invariance assumption in this paper. Methodologically, both papers follow the approach originating in Cox and Huang [3] and Karatzas et al. [17] for the case of additive utility and complete markets, and 
extended in Skiadas [36], Duffie and Skiadas [9], Schroder and Skiadas [30], and El Karoui et al. [10] to recursive utility settings. For a broader context of FBSDE systems in control theory we refer to the books by Ma and Yong [26] and Yong and Zhou [39].

A related literature on constrained portfolio selection with additive utilities $[14,18,35,5,22,16]$ focuses on dual formulations that involve the minimization of the value function over all possible completions of the market. Cuoco [4] showed existence of an optimum under nontradeable income and additive utility with primal methods. We do not discuss duality or existence in this paper. Extensions of the analysis in SS and this paper to nonBrownian settings are developed in Schroder and Skiadas [33].

An alternative to the utility gradient approach, since Merton [27], is dynamic programming. The working paper version of this paper (available at www.kellogg.nwu.edu/faculty/skiadas/research.htm) gives a nonMarkovian dynamic programming optimality verification argument for translationinvariant recursive utility, analogous to the one given in SS for the scale-invariant case. Related Markovian dynamic programming treatments with nontradeable income, additive discounted power utility, and i.i.d. instantaneous returns include Duffie et al. [8] and Koo [21]. The recent additive-exponential-utility solution of Musiela and Zariphopoulou [28] is nested in the setting of this paper, and is extended (in Example 25) by allowing recursive utility and a more general asset structure.

The remainder of the paper is organized in seven sections and two appendices. In Section 2, the market and optimality are defined. In Section 3, optimality is characterized in terms of the relationship between a utility (super)gradient density and a constrained notion of a state-price density. In Section 4, we apply the characterization of optimality to generalized recursive utility, followed by the translation-invariant case in Section 5. In Section 6, the important "quasi-quadratic" specification is introduced, used in applications in the final two sections. Appendix A characterizes aggregators of translation-invariant generalized recursive utilities, and Appendix B contains proofs.

\section{Market and optimality}

Given is an underlying probability space $(\Omega, \mathscr{F}, P)$ supporting a $d$-dimensional standard Brownian motion $B$ over the finite time horizon $[0, T]$. All processes appearing in this paper are assumed to be progressively measurable with respect to the augmented filtration $\left\{\mathscr{F}_{t}: t \in[0, T]\right\}$ generated by $B$. We also assume that $\mathscr{F}_{T}=$ $\mathscr{F}$. The conditional expectation operator $E\left[\cdot \mid \mathscr{F}_{t}\right]$ will be abbreviated to $E_{t}$ throughout. The qualification "almost surely" (or a.s.) will be often omitted where it is implied by the context.

Given any subset $S$ of a Euclidean space, $\mathscr{L}(S)$ denotes the set of all $S$-valued (progressively measurable) processes, and $\mathscr{L}_{p}(S)$ denotes the set of all $x \in \mathscr{L}(S)$ such that $\int_{0}^{T}\left\|x_{t}\right\|^{p} \mathrm{~d} t<\infty$ a.s. (where $\|\cdot\|$ is Euclidean norm). We will make frequent use 
of the spaces:

$$
\begin{aligned}
& \mathscr{S}_{p}=\left\{x \in \mathscr{L}(\mathbb{R}): E\left[\left(\sup _{t \in[0, T]}\left|x_{t}\right|^{p}\right)\right]<\infty\right\}, \\
& \mathscr{H}=\left\{x \in \mathscr{L}(\mathbb{R}): E\left[\int_{0}^{T} x_{t}^{2} \mathrm{~d} t+x_{T}^{2}\right]<\infty\right\} .
\end{aligned}
$$

We consider $\mathscr{H}$ as a Hilbert space under the inner product

$$
(x \mid y)=E\left[\int_{0}^{T} x_{t} y_{t} \mathrm{~d} t+x_{T} y_{T}\right] .
$$

As usual, we identify any elements $x$ and $\tilde{x}$ in $\mathscr{H}$ such that $(x-\tilde{x} \mid x-\tilde{x})=0$.

There is a securities market that allows instantaneous default-free borrowing and lending at a rate given by the stochastic process $r \in \mathscr{L}_{1}(\mathbb{R})$. We refer to trading at this rate as the "money market." The rest of the securities market consists of trading in $m \leqslant d$ risky assets, whose instantaneous excess returns (relative to $r$ ) are represented by the $m$-dimensional Itô process $R$, with dynamics

$$
\mathrm{d} R_{t}=\mu_{t}^{R} \mathrm{~d} t+\sigma_{t}^{R \prime} \mathrm{d} B_{t},
$$

where $\mu^{R} \in \mathscr{L}_{1}\left(\mathbb{R}^{m}\right)$ and $\sigma^{R} \in \mathscr{L}_{2}\left(\mathbb{R}^{d \times m}\right)$. We assume throughout that $\sigma^{R}$ is everywhere full-rank (and therefore everywhere invertible if $m=d$ ).

A trading plan is any process $\phi \in \mathscr{L}\left(\mathbb{R}^{m}\right)$ such that

$$
\int_{0}^{t}\left(\left|\phi_{s}^{\prime} \mu_{s}^{R}\right|+\phi_{s}^{\prime} \sigma_{s}^{R \prime} \sigma_{s}^{R} \phi_{s}\right) \mathrm{d} s<\infty \quad \text { a.s. for all } t<T \text {. }
$$

(All vectors are assumed to be column vectors, with a prime denoting transposition.) We interpret the $i$ th component of $\phi_{t}$ as the time- $t$ market value of the agent's investment in security $i \in\{1, \ldots, m\}$, the remaining wealth being invested in the money market. A (financial) wealth process is any process $W$ such that $W^{-} \in \mathscr{S}_{2}$, where $W_{t}^{-}=\max \left\{0,-W_{t}\right\}$. (This restriction is imposed to rule out doubling-type strategies.) A plan $(c, \phi, W)$ is a triple of a consumption plan $c$, a trading plan $\phi$, and a wealth process $W$.

We consider an agent characterized by the primitives $\left(\mathscr{C}, U_{0}, w_{0}, e, \Phi\right)$. The set $\mathscr{C} \subseteq \mathscr{H}$, whose elements are the consumption plans, is a convex set such that $c+b \in$ $\mathscr{C}$ for any $c \in \mathscr{C}$ and bounded $b \in \mathscr{L}(\mathbb{R})$. Given any $c \in \mathscr{C}$, we think of $c_{t}, t<T$, as the time- $t$ consumption rate and $c_{T}$ as a terminal lump-sum consumption or bequest. The function $U_{0}: \mathscr{C} \rightarrow \mathbb{R}$ is a (strictly) increasing utility function over consumption plans. The positive scalar $w_{0}>0$ is an initial financial wealth, while $e \in \mathscr{H}$ is an endowed income stream, with $e_{t}$ representing a time- $t$ income rate, and $e_{T}$ representing a terminal lump-sum payment. The set $\Phi$, a nonempty convex closed subset of $\mathbb{R}^{m}$, is the agent's constraint set, in which the vector of risky-asset investment values is restricted to lie at all times. (The general analysis would also go through if $\Phi$ were made state and time dependent, subject to some technical measurability conditions.) 
Example 1. Missing market $i$ is modeled by requiring that $\phi^{i}=0$ for all $\phi \in \Phi$. A minimum investment constraint in asset $i$ is modeled by requiring that $\phi^{i} \geqslant l$ for all $\phi \in \Phi$, for some lower limit $l$. If $l=0$, this becomes a short-sale constraint.

The plan $(c, \phi, W)$ is feasible if ${ }^{1}$

$\phi_{t} \in \Phi, \quad t<T$,

and it satisfies the budget equation:

$$
W_{0}=w, \quad \mathrm{~d} W_{t}=\left(W_{t} r_{t}+e_{t}-c_{t}\right) \mathrm{d} t+\phi_{t}^{\prime} \mathrm{d} R_{t}, \quad c_{T}=W_{T}+e_{T} .
$$

A consumption plan $c$ is feasible if $(c, \phi, W)$ is feasible for some trading plan $\phi$ and wealth process $W$. A consumption plan $c$ is optimal if it is feasible and $U(c) \geqslant U(\tilde{c})$ for any other feasible consumption plan $\tilde{c}$, while the plan $(c, \phi, W)$ is optimal if it is feasible and $c$ is optimal. A trading plan $\phi$ is feasible (resp. optimal) if $(c, \phi, W)$ is feasible (resp. optimal) for some $(c, W)$.

\section{Utility gradient and state pricing}

Fixing a reference plan $(c, \phi, W)$, we formulate conditions for its optimality in terms of a utility (super)gradient density and a constrained notion of state pricing. These conditions are applied in subsequent sections in increasingly restrictive settings.

A process $x \in \mathscr{H}$ is a feasible incremental cash flow at $c$ if $c+x$ is a feasible consumption plan. A process $\pi \in \mathscr{H}$ is a state-price density at $c$ if $(\pi \mid x) \leqslant 0$ for every feasible incremental cash flow $x$ at $c$. A process $\pi \in \mathscr{H}$ is a utility supergradient density of $U_{0}$ at $c$ if for all $x \in \mathscr{H}$,

$$
c+x \in \mathscr{C} \text { implies } U_{0}(c+x) \leqslant U_{0}(c)+(\pi \mid x) .
$$

A process $\pi \in \mathscr{H}$ is a utility gradient density of $U_{0}$ at $c$ if for all $x \in \mathscr{H}$,

$$
c+\alpha x \in \mathscr{C} \text { for some } \alpha>0 \text { implies } \quad(\pi \mid x)=\lim _{\alpha \downarrow 0} \frac{U_{0}(c+\alpha x)-U_{0}(c)}{\alpha} .
$$

If $\pi$ is a supergradient density of $U_{0}$ at $c$ and the utility gradient of $U_{0}$ at $c$ exists, then the utility gradient density equals $\pi$.

The supergradient and state-price properties combine to characterize optimality in the following proposition, whose proof is essentially the same as that of Proposition 3 in SS (short for Schroder and Skiadas [32]).

Proposition 2. Suppose $(c, \phi, W)$ is a feasible plan. If $\pi \in \mathscr{H}$ is both a supergradient density of $U_{0}$ at $c$ and a state-price density at $c$, then the plan $(c, \phi, W)$ is optimal. Conversely, if the plan $(c, \phi, W)$ is optimal and $\pi \in \mathscr{H}$ is a utility gradient density of $U_{0}$ at $c$, then $\pi$ is a state-price density at $c$.

\footnotetext{
${ }^{1}$ More precisely, this means that the process $x$ defined by $x_{t}=1\left\{\phi_{t} \notin \Phi\right\}, t<T$, and $x_{T}=0$, is the zero process as an element of $\mathscr{H}$. The analogous interpretation will always be implied for statements of the form " $x_{t} \in S$ " or " $x_{t}=y_{t}$ ".
} 
To apply the last proposition at the reference plan $(c, \phi, W)$, we need to compute the dynamics of the utility gradient (or supergradient) density at $c$, and to develop a criterion for recognizing these dynamics as the dynamics of a state-price density at $c$. Examples of utility (super)gradient calculations appear in Duffie and Skiadas [9], Schroder and Skiadas [30], Chen and Epstein [2], El Karoui et al. [10], as well as in SS. The characterization of state-price dynamics in the current setting is provided in the following result, stated in terms of the support function $\delta_{\Phi}: \mathbb{R}^{m} \rightarrow(-\infty, \infty]$ defined by

$$
\delta_{\Phi}(\varepsilon)=\sup \left\{\phi^{\prime} \varepsilon: \phi \in \Phi\right\} .
$$

Theorem 3. Suppose that $(c, \phi, W)$ is a feasible plan.

(a) (Sufficiency) Suppose that $\pi \in \mathscr{S}_{2}, \pi W \in \mathscr{S}_{1}$, and for some $\eta \in \mathscr{L}_{2}\left(\mathbb{R}^{d}\right)$,

$$
\begin{aligned}
& \frac{\mathrm{d} \pi_{t}}{\pi_{t}}=-r_{t} \mathrm{~d} t-\eta_{t}^{\prime} \mathrm{d} B_{t} \quad \text { for } t \leqslant T, \quad \text { and } \phi_{t}^{\prime} \varepsilon_{t}=\delta_{\Phi}\left(\varepsilon_{t}\right) \quad \text { for } t<T, \\
& \quad \text { where } \varepsilon=\mu^{R}-\sigma^{R} \eta .
\end{aligned}
$$

Then $\pi$ is a state-price density at $c$.

(b) (Necessity) Suppose (for simplicity) that $r$ is bounded, and $\pi \in \mathscr{S}_{1}$ is a strictly positive Itô process. If $\pi$ is a state-price density at $c$, then (4) holds for some $\eta \in$ $\mathscr{L}_{2}\left(\mathbb{R}^{d}\right)$.

By Proposition 2, if $\pi$ is a utility (super)gradient, (4) states the first-order conditions of optimality. In this case we can think of $\pi$ as being the agent's subjective marginal pricing kernel at the optimum, which may not be consistent with the way the market prices assets, due to the agent's constraints. For example, in equilibrium, an agent can regard a stock overvalued if the agent is constrained from short-selling the stock. If $\pi$ follows the dynamics of condition (4), the agent assesses a subjective market-price-of-risk process $\eta$. Given risky asset positions $\phi \in \Phi, \phi^{\prime} \varepsilon$ can be thought of as the instantaneous expected mispricing benefit to the agent, which condition (4) states must be maximized along the optimal path.

The market is complete if $m=d$ and $\Phi=\mathbb{R}^{d}$, in which case there exists a unique, up to positive scaling, state-price density, $\pi$, whose dynamics are given in (4) with $\eta=\left(\sigma^{R \prime}\right)^{-1} \mu^{R}$, provided the process $\pi$ so defined is in $\mathscr{H}$. In the constrained case, the utility gradient density, $\pi$, at the optimum, assuming it exists and is a strictly positive Itô process, defines a market-price-of-risk process $\eta$, which can be interpreted in terms of a fictitious complete market as follows. (Note that the assumption $m=d$ is without loss in generality, since nontradeability of an asset can be modeled through $\Phi$.)

Corollary 4. Suppose that $(c, \phi, W)$ is an optimal plan, $m=d, r$ is bounded, the strictly positive Itô process $\pi \in \mathscr{S}_{2}$ is the gradient density of $U_{0}$ at $c$, and $\pi W \in \mathscr{S}_{1}$. Then there exists $\eta \in \mathscr{L}\left(\mathbb{R}^{d}\right)$ such that condition (4) holds. Moreover, the plan $(c, \phi, W)$ is optimal for the same agent, but with endowed income $e+\delta_{\Phi}(\varepsilon)$ instead of $e$, and no constraints, in a fictitious complete market obtained from the original market by assuming instantaneous expected excess returns $\hat{\mu}^{R}=\sigma^{R \prime} \eta$ instead of $\mu^{R}$. 
Proof. The necessity part of Theorem 3 gives the first-order conditions of optimality in the original market. Feasibility of $(c, \phi, W)$ in the fictitious market is confirmed by direct computation of the budget equation. Finally, optimality in the fictitious market follows by the sufficiency part of Theorem 3 .

\section{Recursive utility}

In this section, we elaborate on the first-order conditions of optimality for the case of generalized recursive utility, by which we mean a utility process that is defined as a solution to a backward stochastic differential equation (BSDE), a continuous-time version of a general backward recursion on an information tree. The specification nests the continuous-time recursive utility of Duffie and Epstein [6], time-additive utility being a special case, the multiple-prior formulation of Chen and Epstein [2], as well as robust-control type criteria through the type of argument in Skiadas [37]. Further properties of generalized recursive utility are discussed in Lazrak and Quenez [23], El Karoui et al. [10], as well as SS. In some cases, ${ }^{2}$ the equivalence in Schroder and Skiadas [31] can be used to mechanically extend the analysis that follows to include linear habit formation.

We define the utility in terms of an aggregator function $F: \Omega \times[0, T] \times \mathbb{R} \times$ $\mathbb{R}^{1+d} \rightarrow \mathbb{R}$, and a set $\mathscr{U} \subseteq \mathscr{S}_{2}$ of utility processes. The random variable $F(T, c, U, \Sigma)$ does not depend on the arguments $(U, \Sigma)$, which are therefore notationally suppressed. We henceforth assume that, given any consumption plan $c$, there is a unique $\left(U, \Sigma^{U}\right) \in \mathscr{U} \times \mathscr{L}_{2}\left(\mathbb{R}^{d}\right)$ that solves the BSDE

$$
\mathrm{d} U_{t}=-F\left(t, c_{t}, U_{t}, \Sigma_{t}^{U}\right) \mathrm{d} t+\Sigma_{t}^{U^{\prime}} \mathrm{d} B_{t}, \quad U_{T}=F\left(T, c_{T}\right),
$$

and we define the utility process of $c$ by letting $U(c)=U$. We also assume that $F(\omega, t, \cdot)$ is a concave function for all $(\omega, t)$, and the derivative, $F_{c}(\omega, t, \cdot, U, \Sigma)$, of $F(\omega, t, \cdot, U, \Sigma)$ exists and maps $\mathbb{R}$ onto $(0, \infty)$.

Example 5 (Duffie-Epstein utility). Duffie and Epstein [6] analyze the aggregator form

$$
F(t, c, U, \Sigma)=f(t, c, U)-\frac{A(t, U)}{2} \Sigma^{\prime} \Sigma,
$$

for some functions $f: \Omega \times[0, T] \times(0, \infty) \times \mathbb{R} \rightarrow \mathbb{R}$ and $A: \Omega \times[0, T] \times \mathbb{R} \rightarrow \mathbb{R}$. The coefficient $A$ can always (under some technical regularity assumptions) be set to zero after a suitable choice of an ordinally equivalent utility. Additive utility is obtained if $f(t, c, U)=u(t, c)-\beta_{t} U$ and $A=0$.

The aggregator $F$ need not be smooth in $(U, \Sigma)$, a generality that is useful, for example, in incorporating the Chen and Epstein [2] formulation. The superdifferential of $F$ with respect to the variables $(U, \Sigma)$ at $(\omega, t, c, U, \Sigma)$ is defined as the

\footnotetext{
${ }^{2}$ For example, this is the case under conical trading constraints if the short rate process and the habit parameters are all deterministic. The equivalence applies generally in the complete-markets case.
} 
set $\left(\partial_{U, \Sigma} F\right)(\omega, t, c, U, \Sigma)$ of all pairs $(a, b) \in \mathbb{R} \times \mathbb{R}^{d}$ such that

$$
F(\omega, t, c+x, U+y, \Sigma+z) \leqslant F(\omega, t, c, U, \Sigma)+F_{c}(\omega, t, c, U, \Sigma) x+a y+b^{\prime} z .
$$

We now fix a reference plan $(c, \phi, W)$ and characterize its optimality. Letting $\left(U, \Sigma^{U}\right)$ be the solution to $\operatorname{BSDE}(5)$, we define the process

$$
\lambda_{t}=F_{c}\left(t, c_{t}, U_{t}, \Sigma_{t}^{U}\right) \text {. }
$$

In a time- $t$ formulation of the agent's problem, $\lambda_{t}$ is the Lagrange multiplier for the time- $t$ wealth constraint, since it provides the first-order utility increment (per unit of wealth) as a result of slightly changing time- $t$ wealth. Consumption can be expressed in terms of $\lambda$ by inverting the above equation: $c_{t}=\mathscr{I}\left(t, \lambda_{t}, U_{t}, \Sigma_{t}\right)$, where the function $\mathscr{I}: \Omega \times[0, T] \times(0, \infty) \times \mathbb{R}^{1+d} \rightarrow \mathscr{C}$ is defined implicitly by

$$
F_{c}(t, \mathscr{I}(t, \lambda, U, \Sigma), U, \Sigma)=\lambda, \quad \lambda \in(0, \infty) .
$$

The utility (super)gradient density can be expressed as a stochastically discounted version of $\lambda$ as follows. Given any processes $(a, b) \in \mathscr{L}_{1}(\mathbb{R}) \times \mathscr{L}_{2}\left(\mathbb{R}^{d}\right)$, the stochastic exponential process $\mathscr{E}(a, b)$ is defined by the $\mathrm{SDE}$

$$
\frac{\mathrm{d} \mathscr{E}_{t}(a, b)}{\mathscr{E}_{t}(a, b)}=a_{t} \mathrm{~d} t+b_{t}^{\prime} \mathrm{d} B_{t}, \quad \mathscr{E}_{0}(a, b)=1 .
$$

Suppose that the process $\pi \in \mathscr{H}$ is given by

$$
\pi_{t}=\mathscr{E}_{t}\left(F_{U}, F_{\Sigma}\right) \lambda_{t}, \quad t \in[0, T],
$$

for some $\left(F_{U}, F_{\Sigma}\right) \in \mathscr{L}_{1}(\mathbb{R}) \times \mathscr{L}_{2}\left(\mathbb{R}^{d}\right)$ such that $\mathscr{E}\left(F_{U}, F_{\Sigma}\right) \in \mathscr{S}_{2}$ and $^{3}$

$$
\left(F_{U}(t), F_{\Sigma}(t)\right) \in\left(\partial_{U, \Sigma} F\right)\left(t, c_{t}, U_{t}, \Sigma_{t}^{U}\right), \quad t \in[0, T] .
$$

Then, by Proposition 13 of SS, $\pi$ is a utility supergradient density of $U_{0}$ at $c$.

Applying Itô's lemma to Eq. (9), condition (4), along with the wealth dynamics and the utility specification results in the following first-order conditions of optimality, to be solved jointly in $\left(U, \Sigma^{U}, \lambda, \sigma^{\lambda}, W\right)$ :

$$
\begin{aligned}
& \mathrm{d} U_{t}=-F\left(t, \mathscr{I}\left(t, \lambda_{t}, U_{t}, \Sigma_{t}^{U}\right), U_{t}, \Sigma_{t}^{U}\right) \mathrm{d} t+\Sigma_{t}^{U \prime} \mathrm{d} B_{t}, \quad U_{T}=F\left(T, W_{T}\right), \\
& \frac{\mathrm{d} \lambda_{t}}{\lambda_{t}}=-\left(r_{t}+F_{U}(t)+\sigma_{t}^{\lambda \prime} F_{\Sigma}(t)\right) \mathrm{d} t+\sigma_{t}^{\lambda \prime} \mathrm{d} B_{t}, \quad \lambda_{T}=F_{c}\left(T, W_{T}\right), \\
& \mathrm{d} W_{t}=\left(W_{t} r_{t}+\phi_{t}^{\prime} \mu_{t}^{R}+e_{t}-\mathscr{I}\left(t, \lambda_{t}, U_{t}, \Sigma_{t}^{U}\right)\right) \mathrm{d} t+\phi_{t}^{\prime} \sigma_{t}^{R^{\prime}} \mathrm{d} B_{t}, \quad W_{0}=w_{0}, \\
& \varepsilon_{t}=\mu_{t}^{R}+\sigma_{t}^{R^{\prime}}\left(F_{\Sigma}(t)+\sigma_{t}^{\lambda}\right), \quad \phi_{t} \in \Phi, \quad \phi_{t}^{\prime} \varepsilon_{t}=\delta_{\Phi}\left(\varepsilon_{t}\right), \\
& \left(F_{U}(t), F_{\Sigma}(t)\right) \in\left(\partial_{U, \Sigma} F\right)\left(t, \mathscr{I}\left(t, \lambda_{t}, U_{t}, \Sigma_{t}^{U}\right), U_{t}, \Sigma_{t}^{U}\right) .
\end{aligned}
$$

The above is a constrained FBSDE. Using last section's results the system is sufficient and necessary for optimality, under suitable regularity assumptions (as given in Proposition 2 and Theorem 3). In a Markovian setting, the above system can be formulated as a PDE system, following the Ma et al. [25] approach. The basic idea is illustrated in SS, and can be easily adapted to this paper's setting.

\footnotetext{
${ }^{3}$ More precisely, we are assuming that the indicator function of $\left(F_{U}(t), F_{\Sigma}(t)\right) \notin\left(\partial_{U, \Sigma} F\right)\left(t, c_{t}, U_{t}, \Sigma_{t}^{U}\right)$ is equal to zero as an element of $\mathscr{H}$.
} 


\section{Translation-invariant formulation}

Having characterized optimality for general recursive utility, in this section we introduce translation invariance relative to a fixed cash flow $\gamma$, a condition that is this paper's main focus. As noted in the Introduction, translationinvariant utility retains the tractability of additive exponential utility without imposing the ad hoc risk-aversion restrictions of additivity. Provided that the cash flow $\gamma$ is traded without constraints, we will see that translation invariance relative to $\gamma$ uncouples the forward and backward components of the first-order conditions, thus reducing last section's FBSDE system to a single BSDE.

For each consumption plan $c$, let $U(c)$ denote the corresponding utility process solving BSDE (5). We fix throughout a bounded process $\gamma \in \mathscr{L}\left(\mathbb{R}_{++}\right)$, and we assume that the dynamic utility $c \mapsto U(c)$ is translation-invariant relative to $\gamma$, meaning that for any $c^{1}, c^{2} \in \mathscr{C}$ and $t \in[0, T]$,

$$
U_{t}\left(c^{1}\right)=U_{t}\left(c^{2}\right) \Rightarrow U_{t}\left(c^{1}+k \gamma\right)=U_{t}\left(c^{2}+k \gamma\right) \quad \text { for all } k \in \mathbb{R} .
$$

Appendix A shows that if we further assume that $U$ is in certainty-equivalent form using $\gamma$ as the numeraire, then $U$ is quasilinear with respect to $\gamma$ :

$$
U_{t}(c+k \gamma)=U_{t}(c)+k \quad \text { for all } k \in \mathbb{R}, c \in \mathscr{C}, t \in[0, T] .
$$

Appendix A also shows that quasilinearity with respect to $\gamma$ is essentially characterized by the aggregator form in the following condition, which is assumed for the remainder of this paper.

Condition 6 (Standing assumption). The following restrictions hold for a bounded process $\gamma \in \mathscr{L}\left(\mathbb{R}_{++}\right)$and a function $G: \Omega \times[0, T] \times \mathbb{R}^{1+d} \rightarrow \mathbb{R}$ that we call an absolute aggregator.

(a) For every $c \in \mathscr{C}, U_{0}(c)=U_{0}$, where $\left(U, \Sigma^{U}\right)$ solves, uniquely in $\mathscr{U} \times \mathscr{L}_{2}\left(\mathbb{R}^{d}\right)$, the BSDE

$$
\mathrm{d} U_{t}=-G\left(t, \frac{c_{t}}{\gamma_{t}}-U_{t}, \Sigma_{t}^{U}\right) \mathrm{d} t+\Sigma_{t}^{U \prime} \mathrm{d} B_{t}, \quad U_{T}=\frac{c_{T}}{\gamma_{T}} .
$$

(b) There exists $\varrho \in \mathbb{R}^{m}$ and a strictly positive process $\Gamma$ such that

$$
\mathrm{d} \Gamma_{t}=\left(r_{t} \Gamma_{t}-\gamma_{t}\right) \mathrm{d} t+\varrho^{\prime} \mathrm{d} R_{t}, \quad \Gamma_{T}=\gamma_{T}
$$

and $v \in \Phi$ implies $v+k \varrho \in \Phi$ for all $k \in \mathbb{R}$.

The notion of an absolute aggregator in a translation-invariant setting is analogous to that of a proportional aggregator in a scale-invariant setting (as explained in Appendix A and SS). Part (b) of the above condition states that the agent can trade with no restrictions in a portfolio $\varrho$ that generates the dividend stream $\gamma$ and has corresponding time- $t$ value $\Gamma_{t}$. Since trading in $\varrho$ is unconstrained, $\delta_{\Phi}(\varepsilon)<\infty$ implies $\varrho^{\prime} \varepsilon=0$. The first-order conditions therefore imply that the agent correctly prices the portfolio $\varrho$. 
Example 7. If $F$ is a Duffie and Epstein [6] aggregator, then the absolute aggregator $G$ must take the functional form

$$
G(\omega, t, x, \Sigma)=g(\omega, t, x)-\frac{A(\omega, t)}{2} \Sigma^{\prime} \Sigma .
$$

In the special case in which

$$
A(\omega, t)=1 \quad \text { and } \quad g(\omega, t, x)=\beta(\omega, t)-\exp (-x),
$$

under suitable integrability restrictions, the ordinally equivalent utility

$$
V_{t}=-\exp \left(-U_{t}\right)
$$

is time-additive discounted exponential utility:

$$
V_{t}=E_{t}\left[\int_{t}^{T}-\exp \left(-\int_{t}^{s} \beta_{u} \mathrm{~d} u-\frac{c_{s}}{\gamma_{s}}\right) \mathrm{d} s-\exp \left(-\int_{t}^{T} \beta_{u} \mathrm{~d} u-\frac{c_{T}}{\gamma_{T}}\right)\right]
$$

The key to simplifying the solution in the translation-invariant case is the following informal argument. Given an optimal plan, suppose that, on some time- $t$ event, $k$ units of account are added to the agent's financial wealth. Because of translation invariance with respect to $\gamma$, the agent will find it optimal to invest all additional $k$ units of wealth in $k / \Gamma_{t}$ shares of the portfolio $\varrho$, and to consume all of the ensuing dividend stream $\left\{k \gamma_{s} / \Gamma_{t} ; s>t\right\}$. Given the quasi-linearity of the utility function with respect to $\gamma$, this suggests the following key relationships at the optimum:

$$
\lambda_{t}=\frac{1}{\Gamma_{t}}, \quad U_{t}=\frac{1}{\Gamma_{t}}\left(Y_{t}+W_{t}\right), \quad \text { and } \quad \phi_{t}=\phi_{t}^{0}+U_{t} \varrho,
$$

where $\left(Y, \Sigma^{Y}, \phi^{0}\right)$ solves a constrained BSDE, given below, that is independent of financial wealth. The second equation states that, taking the market value of the fund $\varrho$ as the unit of account, the value function is the sum of financial wealth and the value of the endowed income stream.

To state the relevant constrained BSDE, we introduce some notation. The (partial) derivative of $G(\omega, t, \cdot, \Sigma)$ at $x$ is denoted $G_{x}(\omega, t, x, \Sigma)$, and the superdifferential of the function $G$ with respect to $\Sigma$ (defined analogously to $\partial_{u, \Sigma} F$ ) is denoted $\partial_{\Sigma} G$. The functions $\mathscr{X}, G^{*}: \Omega \times[0, T] \times(0, \infty) \times \mathbb{R}^{d} \rightarrow \mathbb{R}$ are defined by

$$
\begin{aligned}
G_{x}(\omega, t, \mathscr{X}(\omega, t, y, \Sigma), \Sigma) & =y \\
G^{*}(\omega, t, y, \Sigma) & =\sup _{x \in \mathbb{R}}\{G(\omega, t, x, \Sigma)-y x\} \\
& =G(\omega, t, \mathscr{X}(\omega, t, y, \Sigma), \Sigma)-y \mathscr{X}(\omega, t, y, \Sigma) .
\end{aligned}
$$

Given the conjectured conditions (12), the first-order conditions reduce to a constrained BSDE: 
Condition 8. $\left(Y, \Sigma^{Y}\right) \in \mathscr{L}(\mathbb{R}) \times \mathscr{L}\left(\mathbb{R}^{d}\right)$ and $\phi^{0} \in \mathscr{L}\left(\mathbb{R}^{m}\right)$ solve

$$
\begin{gathered}
\mathrm{d} Y_{t}=\left(Y_{t} r_{t}-\phi_{t}^{0 \prime} \mu_{t}^{R}-e_{t}-\Gamma_{t} G^{*}\left(t, \frac{\gamma_{t}}{\Gamma_{t}}, \frac{\Sigma_{t}^{Y}+\sigma_{t}^{R} \phi_{t}^{0}}{\Gamma_{t}}\right)\right. \\
\left.+\left(\sigma_{t}^{R} \varrho\right)^{\prime}\left(\frac{\sum_{t}^{Y}+\sigma_{t}^{R} \phi_{t}^{0}}{\Gamma_{t}}\right)\right) \mathrm{d} t+\Sigma_{t}^{Y \prime} \mathrm{d} B, \quad Y_{T}=e_{T}, \\
\varepsilon_{t}=\mu_{t}^{R}+\sigma_{t}^{R \prime}\left(G_{\Sigma}(t)-\frac{1}{\Gamma_{t}} \sigma_{t}^{R} \varrho\right), \quad \phi_{t}^{0} \in \Phi, \quad \delta_{\Phi}\left(\varepsilon_{t}\right)=\phi_{t}^{0 \prime} \varepsilon_{t}, \\
G_{\Sigma}(t) \in \partial_{\Sigma} G\left(t, \mathscr{X}\left(t, \frac{\gamma_{t}}{\Gamma_{t}}, \frac{\Sigma_{t}^{Y}+\sigma_{t}^{R} \phi_{t}^{0}}{\Gamma_{t}}\right), \frac{\Sigma_{t}^{Y}+\sigma_{t}^{R} \phi_{t}^{0}}{\Gamma_{t}}\right) .
\end{gathered}
$$

Given $\left(Y, \Sigma^{Y}, \phi^{0}\right)$ and Eqs. (12), the optimal consumption plan is

$$
c_{t}=\gamma_{t}\left(U_{t}+x_{t}\right), \quad \text { where } x_{t}=\mathscr{X}\left(t, \frac{\gamma_{t}}{\Gamma_{t}}, \frac{\Sigma_{t}^{Y}+\sigma_{t}^{R} \phi_{t}^{0}}{\Gamma_{t}}\right) \text {. }
$$

The wealth process dynamics are obtained by substituting the optimal consumption and trading plan expressions in the budget equation:

$$
\begin{aligned}
\mathrm{d} W_{t}= & \left(W_{t} r_{t}+\frac{Y_{t}+W_{t}}{\Gamma_{t}}\left(\varrho^{\prime} \mu_{t}^{R}-\gamma_{t}\right)+\phi_{t}^{0 \prime} \mu_{t}^{R}+e_{t}-\gamma_{t} x_{t}\right) \mathrm{d} t \\
& +\left(\phi_{t}^{0}+\frac{Y_{t}+W_{t}}{\Gamma_{t}} \varrho\right)^{\prime} \sigma_{t}^{R \prime} \mathrm{d} B_{t}, \quad W_{0}=w_{0} .
\end{aligned}
$$

The optimality characterization for the translation-invariant formulation is completed in the following result, proved in Appendix B.

Theorem 9. Suppose Condition 6 holds:

(a) (Sufficiency) Suppose Condition 8 is satisfied, the consumption plan $c$ is defined by Eq. (13), the wealth process $W$ solves $S D E(14)$, and the utility process $U \in \mathscr{U}$ and trading plan $\phi$ are given by Eqs. (12). Finally, suppose $\mathscr{E}=\mathscr{E}\left(-\gamma / \Gamma, G_{\Sigma}\right) \in \mathscr{S}_{2}$, as well as $\mathscr{E} / \Gamma \in \mathscr{S}_{2}$ and $\mathscr{E} W / \Gamma \in \mathscr{S}_{1}$. Then the plan $(c, \phi, W)$ is optimal and $U=U(c)$.

(b) (Necessity) Suppose the plan $(c, \phi, W)$ is optimal, $G(\omega, t, x, \Sigma)$ is differentiable in $(x, \Sigma)$, and $r$ is bounded. Let $U=U(c), x=c / \gamma-U, G_{x}(t)=G_{x}\left(t, x_{t}, \Sigma_{t}^{U}\right)$, and $G_{\Sigma}(t)=G_{\Sigma}\left(t, x_{t}, \Sigma_{t}^{U}\right)$, and suppose that $\mathscr{E}=\mathscr{E}\left(-G_{x}, G_{\Sigma}\right) \in \mathscr{S}_{2}$ and $1 / \Gamma \in \mathscr{S}_{2}$. Then Condition 8 holds with $Y=U \Gamma-W$ and $\phi^{0}=\phi-U \varrho$.

Remark 10. The first-order conditions for the case of no intermediate consumption or the case of no terminal consumption are obtained by omitting the corresponding consumption argument in the utility and supergradient dynamics. The optimality verification argument in those cases remains essentially the same. In the translationinvariant formulation, if there is no intermediate consumption we omit the dependence of $G(\omega, t, x, \Sigma)$ on $x$ and let $\gamma_{t}=0$ for $t<T$, and if there is no terminal consumption we let $U_{T}=\gamma_{T}=0$. 


\section{Quasi-quadratic absolute aggregator}

We have established that translation invariance reduces the FBSDE of the first-order conditions to a single BSDE. To gain further insight into the relationship between trading strategies and risk aversion, in this section we impose a "quasi-quadratic" absolute aggregator functional form. The formulation is sufficiently flexible to model possibly source-dependent first or second-order risk aversion, and includes all translation-invariant Duffie-Epstein utilities, additive discounted exponential utility being a special case. Unlike additive utility, the formulation allows the various types of risk aversion to be arbitrarily selected given the agent's preferences over deterministic plans. The firstorder conditions for a quasi-quadratic absolute aggregator are derived generally in this section, while several more specific solutions are given in the final two sections.

In the remainder of this paper we assume the following condition, using the notation $|x|^{\prime}=\left(\left|x_{1}\right|, \ldots,\left|x_{d}\right|\right), x \in \mathbb{R}^{d}$.

Condition 11 (Standing assumption). The absolute aggregator $G$ is quasi-quadratic, meaning that it takes the form

$$
G(\omega, t, x, \Sigma)=g(\omega, t, x)-q(\omega, t)^{\prime} \Sigma-\kappa(\omega, t)^{\prime}|\Sigma|-\frac{1}{2} \Sigma^{\prime} Q(\omega, t) \Sigma,
$$

for some (progressively measurable) functions $g: \Omega \times[0, T] \times \mathbb{R} \rightarrow \mathbb{R}, q$ : $\Omega \times[0, T] \rightarrow \mathbb{R}^{d}, \kappa: \Omega \times[0, T] \rightarrow \mathbb{R}_{+}^{d}$, and $Q: \Omega \times[0, T] \rightarrow \mathbb{R}^{d \times d}$, such that $Q(\omega, t)$ is symmetric positive definite for all $(\omega, t)$. The processes $\kappa, q$, and $Q$ are assumed bounded (for simplicity).

The interpretation of the various terms of Eq. (15) parallels that of the proportional quasi-quadratic representation in SS, whose Section 5.1 applies in this context with obvious modifications. Assuming it is state independent, the function $g$ entirely determines the agent's preferences over deterministic consumption plans. The linear term $q^{\prime} \Sigma$ can be thought of as reflecting the agent's beliefs, in the sense of a Girsanov change of measure (as explained in SS). The remaining coefficients, $\kappa$ and $Q$, measure, respectively, first and second-order absolute risk aversion that can be time and state dependent, as well as dependent on the source of risk. The sourcedependence of risk aversion can be thought of as reflecting the ambiguity of the risk source in the sense of Ellsberg [11] (see [20] for related ideas). Alternatively, the $\kappa$ term can arise from the Chen and Epstein [2] notion of $\kappa$-ignorance, while the $Q$-term is related to robust control type criteria through the type of argument given in Skiadas [37].

Under a quasi-quadratic absolute aggregator, we obtain the simplifications

$$
\begin{aligned}
& \mathscr{X}(\omega, t, y, \Sigma)=\mathscr{X}^{g}(\omega, t, y), \quad \text { and } \\
& G^{*}(\omega, t, y, \Sigma)=g^{*}(\omega, t, y)-q(\omega, t)^{\prime} \Sigma-\kappa(\omega, t)^{\prime}|\Sigma|-\frac{1}{2} \Sigma^{\prime} Q(\omega, t) \Sigma,
\end{aligned}
$$


where the functions $\mathscr{X}^{g}, g^{*}: \Omega \times[0, T] \times(0, \infty) \rightarrow \mathbb{R}$ are defined analogously to $\mathscr{X}$ and $G^{*}$ by

$$
\begin{aligned}
g_{x}\left(\omega, t, \mathscr{X}^{g}(\omega, t, y)\right) & =y, \\
g^{*}(\omega, t, y) & =\sup _{x \in \mathbb{R}}(g(\omega, t, x)-y x) \\
& =g\left(\omega, t, \mathscr{X}^{g}(\omega, t, y)\right)-y \mathscr{X}^{g}(\omega, t, y) .
\end{aligned}
$$

The superdifferential $\partial_{\Sigma} G$ is characterized as follows. For any $\Sigma \in \mathbb{R}^{d}$, let $\partial|\Sigma|$ denote the set of all $\Lambda \in[-1,1]^{d}$ whose $i$ th coordinate, $\Lambda_{i}$, satisfies

$$
\begin{gathered}
\Lambda_{i}=1 \text { if } \Sigma_{i}>0, \quad \Lambda_{i}=-1 \quad \text { if } \Sigma_{i}<0, \\
\text { and } \Lambda_{i} \in[-1,1] \text { if } \Sigma_{i}=0 .
\end{gathered}
$$

Moreover, for every $x, y \in \mathbb{R}^{d}, x \otimes y \in \mathbb{R}^{d}$ denotes element-by-element multiplication, that is, $(x \otimes y)_{i}=x_{i} y_{i}$ for all $i$. One can easily show:

Lemma 12. $G_{\Sigma}(t) \in\left(\partial_{\Sigma} G\right)\left(t, x_{t}, \Sigma_{t}\right)$ if and only if there exists $\Lambda_{t} \in \partial\left|\Sigma_{t}\right|$ such that $G_{\Sigma}(t)=-\left(q_{t}+\kappa_{t} \otimes \Lambda_{t}+Q_{t} \Sigma_{t}\right)$.

Direct computation using the above lemma gives the constrained BSDE of the first-order conditions as in the following proposition. The term $\kappa \otimes \Lambda$ in the firstorder conditions (which vanishes if the aggregator is smooth) is computed explicitly in the following section assuming that $Q$ is diagonal.

Proposition 13. For the quasi-quadratic absolute aggregator $G$, the constrained BSDE of Condition 8 is equivalent to

$$
\begin{aligned}
& \mathrm{d} Y_{t}=\left(r_{t} Y_{t}-e_{t}-\delta_{\Phi}\left(\varepsilon_{t}\right)-\Gamma_{t} g^{*}\left(t, \frac{\gamma_{t}}{\Gamma_{t}}\right)+\frac{\sum_{t}^{Y \prime} Q_{t} \Sigma_{t}^{Y}-\phi_{t}^{0 \prime} \sigma_{t}^{R \prime} Q_{t} \sigma_{t}^{R} \phi_{t}^{0}}{2 \Gamma_{t}}\right) \mathrm{d} t \\
&+\Sigma_{t}^{Y^{\prime}}\left[\mathrm{d} B_{t}+\left(q_{t}+\kappa_{t} \otimes \Lambda_{t}+\frac{\sigma_{t}^{R} \varrho}{\Gamma_{t}}\right) \mathrm{d} t\right], \quad Y_{T}=e_{T}, \\
& \phi_{t}^{0}= \Gamma_{t}\left(\sigma_{t}^{R \prime} Q_{t} \sigma_{t}^{R}\right)^{-1}\left(\mu_{t}^{R}-\varepsilon_{t}-\sigma_{t}^{R^{\prime}}\left[q_{t}+\kappa_{t} \otimes \Lambda_{t}+\frac{\sigma_{t}^{R} \varrho+Q_{t} \Sigma_{t}^{Y}}{\Gamma_{t}}\right]\right), \\
& \phi_{t}^{0} \in \Phi, \quad \delta_{\Phi}\left(\varepsilon_{t}\right)=\phi_{t}^{0 \prime} \varepsilon_{t}, \quad \Lambda_{t} \in \partial\left|\Sigma_{t}^{Y}+\sigma_{t}^{R} \phi_{t}^{0}\right| .
\end{aligned}
$$

Remark 14. (a) If $\Phi=\mathbb{R}^{m}$, corresponding to possibly incomplete markets (if $m<d$ ) but no further trading constraints, the above first-order conditions simplify by letting $\varepsilon=0$ and $\delta_{\Phi}(\varepsilon)=0$.

(b) If the processes $r, \gamma, \mu^{R}, \sigma^{R}, \kappa, q, Q$, and $e$ are all deterministic, and the function $g$ is state independent, then the first-order conditions simplify by letting $\Sigma^{Y}=0$ and $\varrho=0$.

One can now restate Theorem 9 for the quasi-quadratic case by replacing Condition 8 with the above constrained BSDE. Given a solution to the latter, the complete solution is recovered using Eqs. (12)-(14). 
Example 15. Suppose that the quasi-quadratic absolute aggregator $G$ is smooth (that is, $\kappa=0)$. A simple expression for the optimal trading strategy is obtained under a single linear constraint. Suppose $\Phi=\left\{v \in \mathbb{R}^{m}: l \leqslant p^{\prime} v \leqslant u\right\}$, where $p \in \mathbb{R}^{m}$ and $-\infty \leqslant l \leqslant u \leqslant \infty$. (For example, a short-selling constraint on asset $i$ corresponds to $l=0, u=\infty$, and $p$ a vector with a one in the $i$ th position and zeros elsewhere.) Defining

$$
\phi_{t}^{0 *}=A_{t}\left(\mu_{t}^{R}-\sigma_{t}^{R \prime}\left[q_{t}+\frac{\sigma_{t}^{R} \varrho+Q_{t} \Sigma_{t}^{Y}}{\Gamma_{t}}\right]\right), \quad A_{t}=\Gamma_{t}\left(\sigma_{t}^{R \prime} Q_{t} \sigma_{t}^{R}\right)^{-1},
$$

the pair $\left(\phi^{0}, \varepsilon\right)$ in the first-order conditions of Proposition 13 is given by

$$
\phi_{t}^{0}=\phi_{t}^{0 *}-A_{t} \varepsilon_{t}, \quad \varepsilon_{t}=-\left(p^{\prime} A_{t} p\right)^{-1} p\left(\min \left\{\max \left\{p^{\prime} \phi_{t}^{0 *}, l\right\}, u\right\}-p^{\prime} \phi_{t}^{0 *}\right) .
$$

Note that the above expression for $\phi^{0 *}$ gives the optimal $\phi^{0}$ as a function of $\Sigma^{Y}$ in the unconstrained case. The above claim follows easily from the first-order conditions. The details, as well as the extension to multiple linear constraints, are analogous to Example 34 in SS, and are therefore omitted.

The optimal consumption dynamics for a smooth quasi-quadratic absolute aggregator are summarized in the following proposition, which is followed by an application to equilibrium asset pricing.

Proposition 16. Suppose that Condition 11 holds with $\kappa=0$, the partial $g_{x}$ is state independent, the process $\gamma$ follows the dynamics $\mathrm{d} \gamma / \gamma=\mu^{\gamma} \mathrm{d} t+\sigma^{\gamma \prime} \mathrm{d} B$, and that the first-order conditions of optimality are satisfied at the optimal plan $(c, \phi, W)$, with corresponding utility process $U$. Define

$$
\mathscr{A}_{t}=-\frac{g_{x x}\left(t, x_{t}\right)}{g_{x}\left(t, x_{t}\right)}, \quad \mathscr{S}_{t}=-\frac{g_{x t}\left(t, x_{t}\right)}{g_{x}\left(t, x_{t}\right)}, \quad \mathscr{P}_{t}=-\frac{g_{x x x}\left(t, x_{t}\right)}{g_{x x}\left(t, x_{t}\right)}, \quad x_{t}=\frac{c_{t}}{\gamma_{t}}-U_{t}
$$

and $\sigma_{t}^{\gamma / \Gamma}=\sigma_{t}^{\gamma}-\Gamma_{t}^{-1} \sigma_{t}^{R} \varrho$. Then $\mathrm{d} c_{t}=\mu_{t}^{c} \mathrm{~d} t+\Sigma_{t}^{c \prime} \mathrm{d} B_{t}$, where

$$
\begin{aligned}
\Sigma_{t}^{c}= & \frac{\gamma_{t}}{\Gamma_{t}}\left(\Sigma_{t}^{Y}+\sigma_{t}^{R} \phi_{t}+\left(\frac{1}{\mathscr{A}_{t}}-U_{t}\right) \sigma_{t}^{R} \varrho\right)+\left(c_{t}-\frac{\gamma_{t}}{\mathscr{A}_{t}}\right) \sigma_{t}^{\gamma}, \\
\mu_{t}^{c}= & -\gamma_{t} G\left(t, x_{t}, \Sigma_{t}^{U}\right)+c_{t}\left(\mu_{t}^{\gamma}-\sigma_{t}^{\gamma \prime} \sigma_{t}^{\gamma}\right)+\Sigma_{t}^{c \prime} \sigma_{t}^{\gamma}+\frac{\gamma_{t}}{2} \frac{\mathscr{P}_{t}}{\mathscr{A}_{t}^{2}} \sigma_{t}^{\gamma / \Gamma^{\prime}} \sigma_{t}^{\gamma / \Gamma} \\
& -\frac{\gamma_{t}}{\mathscr{A}_{t}}\left(\mathscr{S}_{t}+\mu_{t}^{\gamma}-r_{t}+\frac{\gamma_{t}}{\Gamma_{t}}-\frac{1}{\Gamma_{t}} \varrho^{\prime}\left(\mu_{t}^{R}+\sigma_{t}^{R \prime} \sigma_{t}^{\gamma / \Gamma}\right)\right) .
\end{aligned}
$$

Example 17 (Equilibrium asset pricing). In addition to the assumptions of the last proposition, we assume that $\Phi=\mathbb{R}^{m}$, meaning that markets can be incomplete $(m<d)$, but there are no further trading constraints. The optimal consumption dynamics can be inverted to obtain the following equilibrium pricing expressions (shown in Appendix B):

$$
\mu_{t}^{R}=\frac{1}{\gamma_{t}} \sigma_{t}^{R^{\prime}}\left[Q_{t} \Sigma_{t}^{c}+\left(\frac{\gamma_{t}}{\mathscr{A}_{t}}-c_{t}\right) Q_{t} \sigma_{t}^{\gamma}+\frac{\gamma_{t}}{\Gamma_{t}}\left(I-\frac{1}{\mathscr{A}_{t}} Q_{t}\right) \sigma_{t}^{R} \varrho\right],
$$




$$
\begin{aligned}
r_{t}= & \mu_{t}^{\gamma}+\frac{\gamma_{t}}{\Gamma_{t}}-\frac{1}{\Gamma_{t}} \varrho^{\prime}\left(\mu_{t}^{R}+\sigma_{t}^{R \prime} \sigma_{t}^{\gamma / \Gamma}\right)+\mathscr{S}_{t}-\frac{\mathscr{P}_{t}}{2 \mathscr{A}_{t}} \sigma_{t}^{\gamma / \Gamma^{\prime}} \sigma_{t}^{\gamma / \Gamma} \\
& +\mathscr{A}_{t}\left[G\left(t, x_{t}, \Sigma_{t}^{U}\right)+\frac{1}{\gamma_{t}}\left(\mu_{t}^{c}+c_{t}\left(\sigma_{t}^{\gamma \prime} \sigma_{t}^{\gamma}-\mu_{t}^{\gamma}\right)-\Sigma_{t}^{c \prime} \sigma_{t}^{\gamma}\right)\right] .
\end{aligned}
$$

For example, if $g(\omega, t, x)=\beta(\omega, t)-\exp (-a x), a \in \mathbb{R}_{++}$, the above expressions simplify since $\mathscr{A}_{t}=\mathscr{P}_{t}=a$ and $\mathscr{S}_{t}=0$. If markets are complete, or preferences are Duffie-Epstein (that is, $Q=b I, b \in \mathscr{L}\left(\mathbb{R}_{++}\right)$), the risk-premium equilibrium expression can be added-up across agents in an economy with agent-specific parameters $(a, b, \beta)$, to obtain an equilibrium expression for $\mu^{R}$ in terms of the aggregate endowment and its volatility.

\section{Solutions under quasi-quadratic aggregator}

This section presents more detailed solutions under a quasi-quadratic absolute aggregator, including an explicit expression for the term $\kappa \otimes \Lambda$ in the first-order conditions given diagonal $Q$, clarifying the role of first-order risk aversion, as well as some special solutions emphasizing the effect of undiversifiable income.

The following normalization of the return volatility process $\sigma^{R}$ and the secondorder risk aversion parameter $Q$ will be henceforth assumed. The restriction is vacuous if $m=d$, which can be assumed without loss in generality by modeling missing markets through $\Phi$. In applications, however, it is often more convenient to assume $m<d$.

Condition 18 (Standing assumption). For some $\sigma_{M M}^{R}, Q_{M M} \in \mathscr{L}\left(\mathbb{R}^{m \times m}\right)$,

$$
\sigma^{R}=\left[\begin{array}{l}
\sigma_{M M}^{R} \\
0
\end{array}\right] \text { and } Q=\left[\begin{array}{cc}
Q_{M M} & 0 \\
0 & Q_{N N}
\end{array}\right] \text {. }
$$

Remark 19. By Lemma 10 of SS, $\sigma^{R}$ can always be put in the above form by passing to a new Brownian motion that generates the same filtration as the original one. Suppose $Q=a I$ for some $a \in \mathbb{R}$ (where $I$ denotes the identity matrix), as is the case with Duffie-Epstein utility, including time-additive utility. Then the change of Brownian motion does not affect $Q$, and therefore imposing Condition 18 entails no loss in generality (even if $m<d$ ).

The notational scheme suggested in the above condition will be followed throughout: $M$ and $N$ refer to the sets of marketed and nonmarketed securities, respectively, and are also used as indices of corresponding matrix blocks. In particular, given any $d$-dimensional process or vector $x$, we write $x^{\prime}=\left[x_{M}^{\prime}, x_{N}^{\prime}\right]$, where $x_{M}$ is valued in $\mathbb{R}^{m}$ and $x_{N}$ is valued in $\mathbb{R}^{d-m}$. Given the new subscripts, we often omit dummy time indices to simplify the notation. For example, the above condition implies that $\mathrm{d} R=\mu^{R} \mathrm{~d} t+\sigma_{M M}^{R \prime} \mathrm{d} B_{M}$. It is important to note that, unless otherwise stated, the coefficients $\mu^{R}$ and $\sigma_{M M}^{R}$ are not required to be adapted to the filtration generated by $B_{M}$. 
The following processes will be of repeated use:

$$
\eta_{M}=\left(\sigma_{M M}^{R \prime}\right)^{-1}\left(\mu^{R}-\varepsilon\right) \text { and } \quad \alpha_{M}=\eta_{M}-q_{M}-\frac{1}{\Gamma} \sigma_{M M}^{R} \varrho .
$$

Moreover, in the first-order conditions, we will often refer to the term

$$
\hat{\alpha}_{M}=\alpha_{M}-\kappa_{M} \otimes \Lambda_{M} .
$$

For example, in this context, the optimal trading strategy is $\phi=\phi^{0}+U \varrho$, where

$$
\phi^{0}=\left(\sigma_{M M}^{R}\right)^{-1}\left(\Gamma Q_{M M}^{-1} \hat{\alpha}_{M}-\Sigma_{M}^{Y}\right) .
$$

Example 20 (Fictitious completion). This example illustrates Corollary 4. Suppose that $m<d$ and $\Phi=\mathbb{R}^{m}$. The fictitious complete market of Corollary 4 is obtained by introducing unrestricted trading in $d-m$ fictitious securities with price dynamics specified by $\mu_{N}^{R}=q_{N}+\kappa_{N} \otimes \Lambda_{N}+\Gamma^{-1} Q_{N N} \Sigma_{N}^{Y}, \sigma_{N N}^{R}$ equal to the identity matrix, and $\sigma_{M N}^{R}=\sigma_{N M}^{R \prime}=0$. The agent's endowment and the marketed asset price dynamics are the same in the original and fictitious markets. The first-order conditions in the fictitious market imply that $\phi_{N}=0$; and therefore the impossibility of trading in fictitious assets is a nonbinding constraint. Direct computation shows that the BSDE for $Y$ is the same in the fictitious market and the original market.

The following result clarifies the role of $\kappa$ in the first-order conditions, given a diagonal process $Q$.

Proposition 21. Suppose Condition 18 holds, $Q$ is diagonal, and the process $\alpha_{M}$ is defined in (16). Then, in the first-order conditions of Proposition 13, the terms $\kappa \otimes \Lambda$ and $\phi^{0}$ can equivalently be specified by

$$
\kappa_{M} \otimes \Lambda_{M}=\max \left\{\min \left\{\alpha_{M}, \kappa_{M}\right\},-\kappa_{M}\right\}, \quad \kappa_{N} \otimes \Lambda_{N}=\kappa_{N} \otimes \operatorname{sign}\left(\Sigma_{N}^{Y}\right),
$$

and Eq. (18), where $\hat{\alpha}_{M}$ can be written (equivalently to (17)) as

$$
\hat{\alpha}_{M}=\min \left\{\max \left\{0, \alpha_{M}-\kappa_{M}\right\}, \alpha_{M}+\kappa_{M}\right\}=Q_{M M} \Sigma_{M}^{U} .
$$

Given the assumption that $Q$ is diagonal, the last equation implies that, for any $i \in M, \Sigma_{i}^{U}=0$ whenever $\left|\alpha_{i}\right| \leqslant \kappa_{i}$; that is, the agent completely hedges the $i$ th dimension of risk, as a consequence of first-order risk aversion. Analogous results for the scale-invariant case are presented in SS (see also [34,2,13]).

Example 22. Suppose further that the parameters $\left(r, \gamma, \mu^{R}, \sigma^{R}, \kappa, q, Q, e\right)$ are all deterministic, and the function $g$ is state independent. Then $Y$ is also deterministic, and therefore $\Sigma^{Y}=0$ and $\phi^{0}=\left(\sigma_{M M}^{R}\right)^{-1} \Gamma \Sigma_{M}^{U}$. In this case, $\Sigma_{i}^{U}=0$ can imply complete nonparticipation in the market for asset $i$. For example, suppose that $\sigma_{M M}^{R}$ (as well as $Q$ ) is diagonal, and $\Phi=\mathbb{R}^{m}$. Suppose also that the portfolio $\varrho$ consists of a share in asset one, generating dividend stream $\gamma$. Then, for any $i \geqslant 2$, $\left|\left(\mu_{i}^{R} / \sigma_{i i}^{R}\right)-q_{i}\right|$ $\leqslant \kappa_{i}$ implies $\phi_{i}=0$. That is, a sufficiently small belief-adjusted market price of the risk traded by asset $i \geqslant 2$ results in the agent's nonparticipation in this market. 
This section's last main result focuses on the role of a nontradeable endowment income stream. We assume that $\Phi=\mathbb{R}^{m}$, which implies that markets can be incomplete $(m<d)$, but there are no further trading constraints. Assuming that all parameters, except for the endowment $e$, depend only on uncertainty generated by $B_{M}$, we derive a decomposition of $Y$ into a subjective endowment value component and a market component.

Proposition 23. Suppose that $\Phi=\mathbb{R}^{m}$, and the processes $r, \gamma, \mu^{R}, \sigma^{R}, \kappa_{M}, q_{M}, Q_{M M}$, and $g^{*}(\gamma / \Gamma)$ are all adapted to the (augmented) filtration $\left\{\mathscr{F}_{t}^{M}\right\}$ generated by $B_{M}$. Then, in the first-order conditions of Proposition 13, the process $Y$ and its volatility can be decomposed as

$$
Y=Y^{M}+Y^{e}, \quad \Sigma_{M}^{Y}=\Sigma_{M}^{Y^{M}}+\Sigma_{M}^{Y^{e}}, \quad \text { and } \quad \Sigma_{N}^{Y}=\Sigma_{N}^{Y^{e}},
$$

where $\left(Y^{M}, \Sigma_{M}^{Y^{M}}\right)$ is adapted to $\left\{\mathscr{F}_{t}^{M}\right\}$ and solves the BSDE:

$$
\begin{aligned}
\mathrm{d} Y^{M}= & -\left(\Gamma g^{*}\left(\frac{\gamma}{\Gamma}\right)-r Y^{M}+\frac{\Gamma}{2} \hat{\alpha}_{M}^{\prime} Q_{M M}^{-1} \hat{\alpha}_{M}\right) \mathrm{d} t \\
& +\Sigma_{M}^{Y^{M}}\left(\mathrm{~d} B_{M}+\eta_{M} \mathrm{~d} t\right), \quad Y_{T}^{M}=0,
\end{aligned}
$$

while $\left(Y^{e}, \Sigma^{Y^{e}}\right)$ is adapted to the finer filtration $\left\{\mathscr{F}_{t}\right\}$ and solves the BSDE:

$$
\begin{aligned}
\mathrm{d} Y^{e}= & -\left(e-r Y^{e}-\frac{1}{2 \Gamma} \Sigma_{N}^{Y^{e}{ }^{\prime}} Q_{N N} \Sigma_{N}^{Y^{e}}\right) \mathrm{d} t \\
& +\Sigma_{M}^{Y^{e}}\left(\mathrm{~d} B_{M}+\eta_{M} \mathrm{~d} t\right)+\Sigma_{N}^{Y^{e}}\left(\mathrm{~d} B_{N}+\left(q_{N}+\kappa_{N} \otimes \Lambda_{N}\right) \mathrm{d} t\right), \quad Y_{T}^{e}=e_{T} .
\end{aligned}
$$

To present integrated versions of the above BSDEs, we introduce some notation with regard to Girsanov changes of measure. Given any $b \in \mathscr{L}\left(\mathbb{R}^{d}\right)$, we use the notation $B^{b}$ and $\xi^{b}$ to denote the processes satisfying

$$
\mathrm{d} B_{t}^{b}=\mathrm{d} B_{t}+b_{t} \mathrm{~d} t, \quad B_{0}^{b}=0, \quad \text { and } \quad \frac{\mathrm{d} \xi_{t}^{b}}{\xi_{t}^{b}}=-b_{t}^{\prime} \mathrm{d} B_{t}, \quad \xi_{0}^{b}=1 .
$$

If $\xi^{b}$ is a martingale (for example, if $b$ satisfies the Novikov condition), then $B^{b}$ is Brownian motion under the probability $P^{b}$ with density $\mathrm{d} P^{b} / \mathrm{d} P=\xi_{T}^{b}$. The expectation operator corresponding to the probability $P^{b}$ is denoted $E^{b}$.

Consider now the processes

$$
\theta=\left[\begin{array}{c}
\eta_{M} \\
q_{N}+\kappa_{N} \otimes \Lambda_{N}
\end{array}\right] \text { and } \mathscr{B}_{t, s}=\exp \left(-\int_{t}^{s} r_{u} \mathrm{~d} u\right)
$$

and assume that $\xi^{\theta}$ is a martingale. The above BSDEs can then be integrated, provided the integrals below are well-defined, to obtain the following expressions for the two components of $Y$ :

$$
\begin{gathered}
Y_{t}^{M}=E^{\theta}\left[\int_{t}^{T} \mathscr{B}_{t, s} \Gamma_{s}\left(g^{*}\left(s, \frac{\gamma_{s}}{\Gamma_{s}}\right)+\frac{1}{2} \hat{\alpha}_{M}^{\prime}(s) Q_{M M}^{-1}(s) \hat{\alpha}_{M}(s)\right) \mathrm{d} s \mid \mathscr{F}_{t}^{M}\right], \\
Y_{t}^{e}=E^{\theta}\left[\int_{t}^{T} \mathscr{B}_{t, s}\left(e_{s}-\frac{1}{2 \Gamma_{s}} \Sigma_{N}^{e \prime}(s) Q_{N N}(s) \Sigma_{N}^{e}(s)\right) \mathrm{d} s+\mathscr{B}_{t, T} e_{T} \mid \mathscr{F}_{t}\right] .
\end{gathered}
$$


Example 24 (Complete markets). Suppose that markets are complete, that is, $m=d$ and $\Phi=\mathbb{R}^{m}$. Then $\eta=\left(\sigma^{R \prime}\right)^{-1} \mu^{R}$ is the unique market price of risk process, and $Y_{t}^{e}=E_{t}^{\eta}\left[\int_{t}^{T} \mathscr{B}_{t, s} e_{s} \mathrm{~d} s+\mathscr{B}_{t, T} e_{T}\right]$ is the time- $t$ market value of the endowment.

Example 25. We consider two special cases of Proposition 23 in which the subjective value of the endowment, $Y^{e}$, is given by an expression of the form

$$
\exp \left(-\frac{a}{\Gamma_{t}} Y_{t}^{e}\right)=E_{t}^{b}\left[\exp \left(-\int_{t}^{T} \frac{a}{\Gamma_{s}} e_{s} \mathrm{~d} s-\frac{a}{\Gamma_{T}} e_{T}\right)\right]
$$

for properly defined $a$ and $b$, extending a more special result by Musiela and Zariphopoulou [28]. The proofs of the following claims are given in Appendix B. In addition to the assumptions of Proposition 23, we assume that there is no intermediate consumption (meaning that for every $c \in \mathscr{C}, c_{t}=0$ for $t<T$ ), and in particular $\gamma_{t}=0$ for all $t<T$ (which presents no problems since the strict positivity of $\gamma_{T}$ implies that of $\Gamma$ ). Even though we have not treated the case of no-intermediate consumption separately, the results are essentially the same (see Remark 10). We also assume that $r$ and $\gamma_{T}>0$ are deterministic, and that $\kappa_{N}=0$. Within this setting we consider two special cases:

Case 1 (idiosyncratic endowment): Suppose that $e$ is adapted to the filtration generated by $B_{N}$, and $Q_{N N}(t)=a I$, where $a>0$ and $I$ is an identity matrix. Then $\Sigma_{M}^{Y^{e}}=0$ and Eq. (20) holds for any $b$ such that $b_{N}=q_{N}$, provided $\xi^{b}$ is a martingale and the stated integral exists.

Case 2 (nonidiosyncratic endowment): Suppose that $Q_{N N}$ is constant over time; $\eta_{M}$ and $q_{N}$ are deterministic; and the endowment process satisfies

$$
\mathrm{d} e_{t}=\mu_{t} \mathrm{~d} t+\beta_{t} \Sigma^{\prime} \mathrm{d} B_{t},
$$

where $\Sigma \in \mathbb{R}^{d}$, and $\mu$ and $\beta$ are real-valued and adapted to the filtration generated by $e$. Then Eq. (20) holds with $a=\Sigma_{N}^{\prime} Q_{N N} \Sigma_{N} / \Sigma^{\prime} \Sigma$ and $b^{\prime}=\theta^{\prime}=\left[\eta_{M}^{\prime}, q_{N}^{\prime}\right]$. The solution of Musiela and Zariphopoulou [28] is the subcase obtained by assuming $Q=I$ (timeadditive utility), $q=0$, Markovian dynamics for $e$ and $R$, and a single lognormally distributed risky asset.

\section{Incomplete markets and quadratic BSDEs}

In incomplete but otherwise unconstrained markets, and under a quasi-quadratic smooth absolute aggregator, the BSDE of the first-order conditions characterizing $Y$ takes a quadratic form. A quadratic BSDE is also obtained in SS in a scale-invariant setting with different preference restrictions. As in SS, we summarize below a set of conditions under which the quadratic BSDE can be reduced to a system of ordinary differential equations (ODEs), leading to some new tractable applications with nontradeable income. The technique can be applied to BSDEs as well as the corresponding PDEs in a Markovian setting. The PDE version of the approach is familiar from the affine term-structure literature (see [7,29]), and has been utilized in scale-invariant consumption/portfolio problems (subsumed by the formulation of 
SS) by Kim and Omberg [19], Chacko and Viceira [1], Schroder and Skiadas [30], and Liu [24].

The following restrictions are imposed throughout this subsection:

Condition 26. Markets can be incomplete but there are no other constraints $(\Phi=$ $\left.\mathbb{R}^{m}\right)$, the absolute aggregator is smooth $(\kappa=0)$, and $q=0$.

The assumption $q=0$ is without loss of generality (via a Girsanov change-ofmeasure argument). Under these assumptions, the BSDE characterizing $Y$ in the first-order conditions of Proposition 13 becomes

$$
\mathrm{d} Y_{t}=-\left(e_{t}+p_{t}-r_{t} Y_{t}+\Sigma_{t}^{Y \prime} h_{t}+\frac{1}{2} \Sigma_{t}^{Y \prime} H_{t} \Sigma_{t}^{Y}\right) \mathrm{d} t+\Sigma_{t}^{Y \prime} \mathrm{d} B_{t}, \quad Y_{T}=e_{T},
$$

where

$$
\begin{aligned}
& p_{t}=\Gamma_{t} g^{*}\left(t, \frac{\gamma_{t}}{\Gamma_{t}}\right)+\frac{\Gamma_{t}}{2}\left(\mu_{t}^{R}-\frac{1}{\Gamma_{t}} \sigma_{t}^{R^{\prime}} \sigma_{t}^{R} \varrho\right)^{\prime}\left(\sigma_{t}^{R \prime} Q_{t} \sigma_{t}^{R}\right)^{-1}\left(\mu_{t}^{R}-\frac{1}{\Gamma_{t}} \sigma_{t}^{R^{\prime}} \sigma_{t}^{R} \varrho\right), \\
& h_{t}=-\frac{\sigma_{t}^{R} \varrho}{\Gamma_{t}}-Q_{t} \sigma_{t}^{R}\left(\sigma_{t}^{R \prime} Q_{t} \sigma_{t}^{R}\right)^{-1}\left(\mu_{t}^{R}-\frac{1}{\Gamma_{t}} \sigma_{t}^{R \prime} \sigma_{t}^{R} \varrho\right), \text { and } \\
& H_{t}=\frac{1}{\Gamma_{t}}\left(Q_{t} \sigma^{R}\left(\sigma_{t}^{R \prime} Q_{t} \sigma_{t}^{R}\right)^{-1} \sigma^{R^{\prime}} Q_{t}-Q_{t}\right) .
\end{aligned}
$$

We now formulate a set of conditions that reduce BSDE (21) to an ODE system. We introduce a state process $Z \in \mathscr{L}\left(\mathbb{R}^{k}\right)$ with dynamics

$$
\mathrm{d} Z_{t}=\mu_{t}^{Z} \mathrm{~d} t+\sigma_{t}^{Z \prime} \mathrm{d} B_{t}, \quad \text { where } \mu^{Z} \in \mathscr{L}_{1}\left(\mathbb{R}^{k}\right), \sigma^{Z} \in \mathscr{L}_{2}\left(\mathbb{R}^{d \times k}\right) .
$$

Moreover, we split these state variables into two blocks, of dimensionality $a$ and $b$, respectively, where $a+b=k$. We treat $a$ and $b$ as integers denoting dimensionality, as well as indices of corresponding matrix blocks, writing

$$
Z=\left[\begin{array}{l}
Z^{a} \\
Z^{b}
\end{array}\right] \text { and } \sigma^{Z}=\left[\begin{array}{ll}
\sigma^{Z a} & \sigma^{Z b}
\end{array}\right]
$$

where $Z^{a} \in \mathscr{L}\left(\mathbb{R}^{a}\right), Z^{b} \in \mathscr{L}\left(\mathbb{R}^{b}\right), \sigma^{Z a} \in \mathscr{L}\left(\mathbb{R}^{d \times a}\right)$, and $\sigma^{Z b} \in \mathscr{L}\left(\mathbb{R}^{d \times b}\right)$.

We seek a solution of the form

$$
Y_{t}=Y_{0}(t)+Y_{1}(t)^{\prime} Z_{t}+\frac{1}{2} Z_{t}^{a \prime} Y_{2}(t) Z_{t}^{a}
$$

for some deterministic processes $Y_{0} \in \mathscr{L}(\mathbb{R}), Y_{1}=\left[Y_{1}^{a \prime}, Y_{1}^{b^{\prime}}\right]^{\prime} \in \mathscr{L}\left(\mathbb{R}^{k}\right)$, and $Y_{2} \in$ $\mathscr{L}\left(\mathbb{R}^{a \times a}\right)$. A sufficient set of conditions for this type of solution is stated below (omitting time indices). We assume, without loss of generality, that all matrices appearing in quadratic forms, including $Y_{2}(t)$ above, are symmetric.

Condition 27. (a) The processes $r$ and $\sigma^{Z a}$ are deterministic.

(b) For some deterministic processes $J_{0}, K_{0} \in \mathscr{L}(\mathbb{R}) ; J_{1}, K_{1} \in \mathscr{L}\left(\mathbb{R}^{k}\right)$; and $J_{2}, K_{2} \in$ $\mathscr{L}\left(\mathbb{R}^{a \times a}\right)$,

$$
e=J_{0}+J_{1}^{\prime} Z+\frac{1}{2} Z^{a \prime} J_{2} Z^{a} \text { and } p=K_{0}+K_{1}^{\prime} Z+\frac{1}{2} Z^{a \prime} K_{2} Z^{a} .
$$


(c) For some deterministic processes $L_{0}=\left[L_{0}^{a \prime}, L_{0}^{b \prime}\right]^{\prime} \in \mathscr{L}\left(\mathbb{R}^{k}\right), L_{1}^{a} \in \mathscr{L}\left(\mathbb{R}^{a \times a}\right), L_{1}^{b} \in$ $\mathscr{L}\left(\mathbb{R}^{b \times k}\right)$, and $L_{2}^{b}[i] \in \mathscr{L}\left(\mathbb{R}^{a \times a}\right)$,

$$
\mu^{Z}+\sigma^{Z \prime} h=L_{0}+\left(\begin{array}{c}
L_{1}^{a} Z^{a} \\
L_{1}^{b} Z+\left[Z^{a \prime} L_{2}^{b}[i] Z^{a}\right]_{i=1, \ldots, b}
\end{array}\right) .
$$

(d) For some deterministic processes $D_{0}^{a a} \in \mathscr{L}\left(\mathbb{R}^{a \times a}\right), \quad D_{0}^{a b} \in \mathscr{L}\left(\mathbb{R}^{a \times b}\right), \quad D_{0}^{b b} \in$ $\mathscr{L}\left(\mathbb{R}^{b \times b}\right), D_{1}^{a b}[i, j] \in \mathscr{L}\left(\mathbb{R}^{a}\right), D_{1}^{b b}[i, j] \in \mathbb{R}^{k}$ and $D_{2}^{b b}[i, j] \in \mathscr{L}\left(\mathbb{R}^{a \times a}\right)$,

$$
\begin{aligned}
\sigma^{Z \prime} H \sigma^{Z}= & \left(\begin{array}{cc}
D_{0}^{a a} & D_{0}^{a b} \\
D_{0}^{a b \prime} & D_{0}^{b b}
\end{array}\right) \\
& +\left(\begin{array}{cc}
0 & {\left[Z^{a \prime} D_{1}^{a b}[i, j]\right]_{i=1, \ldots, a ; j=1, \ldots, b}} \\
{\left[Z^{a \prime} D_{1}^{a b}[i, j]\right]_{i=1, \ldots, a, j=1, \ldots, b}^{\prime}} & {\left[Z^{\prime} D_{1}^{b b}[i, j]\right]_{i, j=1, \ldots, b}}
\end{array}\right) \\
& +\left(\begin{array}{cc}
0 & 0 \\
0 & {\left[Z^{a \prime} D_{2}^{b b}[i, j] Z^{a}\right]_{i, j=1, \ldots, b}}
\end{array}\right) .
\end{aligned}
$$

We let $D_{0} \in \mathscr{L}\left(\mathbb{R}^{k \times k}\right)$ denote the first term of the right-hand side.

Suppose Conditions 26 and 27 hold, $\Xi \in \mathscr{L}\left(\mathbb{R}^{a \times a}\right)$ is defined to have $i$ th row

$$
\Xi_{i *}=\sum_{j=1}^{b} Y_{1 j}^{b} D_{1}^{a b}[i, j]^{\prime}
$$

and the deterministic processes $\left(Y_{0}, Y_{1}, Y_{2}\right)$ solve the following ODE system (where the left-hand sides denote time-derivatives):

$$
\begin{aligned}
\dot{Y}_{0}= & r Y_{0}-J_{0}-K_{0}-Y_{1}^{\prime} L_{0}-\frac{1}{2} Y_{1}^{\prime} D_{0} Y_{1}-\frac{1}{2} \operatorname{trace}\left(Y_{2} \sigma^{Z a \prime} \sigma^{Z a}\right), \\
\dot{Y}_{1}= & r Y_{1}-J_{1}-K_{1}-L_{1}^{b \prime} Y_{1}^{b}-\frac{1}{2} \sum_{i=1}^{b} \sum_{j=1}^{b} Y_{1 i}^{b} Y_{1 j}^{b} D_{1}^{b b}[i, j] \\
& -\left(\begin{array}{ll}
\left.L_{1}^{a \prime} Y_{1}^{a}+\sum_{i=1}^{a} \sum_{j=1}^{b} Y_{1 i}^{a} Y_{1 j}^{b} D_{1}^{a b}[i, j]+Y_{2}\left(D_{0}^{a a} \quad D_{0}^{a b}\right) Y_{1}+Y_{2} L_{0}^{a}\right), \\
0
\end{array}\right. \\
\dot{Y}_{2}= & r Y_{2}-J_{2}-K_{2}-Y_{2} L_{1}^{a}-L_{1}^{a \prime} Y_{2}-Y_{2} D_{0}^{a a} Y_{2}-Y_{2} \Xi-\Xi^{\prime} Y_{2} \\
& -\sum_{i=1}^{b} \sum_{j=1}^{b} Y_{1 i}^{b} Y_{1 j}^{b} D_{2}^{b b}[i, j]-2 \sum_{i=1}^{b} Y_{1 i}^{b} L_{2}^{b}[i],
\end{aligned}
$$

with terminal conditions $Y_{i}(T)=J_{i}(T), i=0,1,2$.

Direct computation using Itô's lemma shows that then Eq. (22) defines a solution to BSDE (21) (provided that the drift and diffusion terms of (21) are suitably integrable so that the respective integrals are well-defined).

Example 28. We assume that Condition 26 is satisfied, that the processes $Q, r$, and $\gamma$ are deterministic, that $g^{*}$ is state independent, and (without loss of generality) that 
$\mathrm{d} R=\mu^{R} \mathrm{~d} t+\sigma_{M M}^{R \prime} \mathrm{d} B_{M}$. These assumptions imply that $\varrho=0$, and that $\Gamma_{t}$ and $g^{*}\left(t, \gamma_{t} / \Gamma_{t}\right)$ are also deterministic. We also recall the price of marketed risk expression $\eta_{M}=\left(\sigma_{M M}^{R \prime}\right)^{-1} \mu^{R}$. The following two problem classes satisfy Condition 27.

Class 1: $Z=Z^{a}(a=k, b=0)$, and

$$
\begin{aligned}
& \mathrm{d} Z_{t}=\left(\mu-\beta Z_{t}\right) \mathrm{d} t+\Sigma^{\prime} \mathrm{d} B_{t}, \\
& \eta_{M}=v+V Z_{t}, \quad e_{t}=J_{0}+J_{1} Z_{t}+\frac{1}{2} Z_{t}^{\prime} J_{2} Z_{t},
\end{aligned}
$$

where $\mu \in \mathbb{R}^{a}, \Sigma \in \mathbb{R}^{d \times a}, \beta \in \mathbb{R}^{a \times a}, v \in \mathbb{R}^{m}, V \in \mathbb{R}^{m \times a}, J_{0} \in \mathbb{R}, J_{1} \in \mathbb{R}^{a}$, and $J_{2} \in$ $\mathbb{R}^{a \times a}$.

Class 2: $Z=Z^{b}(a=0, b=k), Q$ satisfies Condition 18 with $Q_{N N}=\gamma I$ for some positive constant $\gamma$, and

$$
\begin{aligned}
& \mathrm{d} Z_{t}=\left(\mu-\beta Z_{t}\right) \mathrm{d} t+\Sigma^{\prime} \operatorname{diag}\left(\sqrt{v+V Z_{t}}\right) \mathrm{d} B_{t}, \\
& \eta_{M}=\operatorname{diag}\left(\sqrt{v_{M}+V_{M *} Z_{t}}\right) \varphi, \quad e_{t}=J_{0}+J_{1}^{\prime} Z_{t},
\end{aligned}
$$

where $\operatorname{diag}(x)$ denotes the diagonal matrix with $x$ on the diagonal, $\sqrt{x}$ denotes the vector with ith element $\sqrt{x_{i}}$, and $\mu \in \mathbb{R}^{b}, \Sigma \in \mathbb{R}^{d \times b}, \beta \in \mathbb{R}^{b \times b}, v=\left[v_{M}^{\prime}, v_{N}^{\prime}\right]^{\prime} \in \mathbb{R}^{d}$, $V=\left[V_{M *}^{\prime}, V_{N *}^{\prime}\right]^{\prime} \in \mathbb{R}^{d \times b}, \varphi \in \mathbb{R}^{m}, J_{0} \in \mathbb{R}, J_{1} \in \mathbb{R}^{b}$. In this case, $Y_{2}=0$, and only the first two Riccati equations are needed, the second unlinked to the first. This formulation includes the square-root volatility specification of Heston [15].

\section{Acknowledgement}

We thank Ali Lazrak for his useful discussion of this paper.

\section{Appendix A. Translation-invariant recursive utility representation}

In this appendix, we argue the necessity of the functional form of Condition 6 for translation invariance relative to $\gamma$. We consider the dynamic utility that assigns to each consumption plan $c$ a utility process $U(c)$ that is the unique process in $\mathscr{U}$ solving BSDE (5). We fix a bounded process $\gamma \in \mathscr{L}\left(\mathbb{R}_{++}\right)$throughout, and we assume that for every $U \in \mathscr{U}$ and time $t$, there exists a consumption plan $c$ such that $c_{s}=U_{t} \gamma_{s}$ for all $s \geqslant t$. We also recall that the dynamic utility $c \mapsto U(c)$ is translation-invariant relative to $\gamma$, or $\gamma-T I$ for short, if for any $k \in \mathbb{R}, c^{1}, c^{2} \in \mathscr{C}$, and $t \in[0, T], U_{t}\left(c^{1}\right)=$ $U_{t}\left(c^{2}\right)$ implies $U_{t}\left(c^{1}+k \gamma\right)=U_{t}\left(c^{2}+k \gamma\right)$.

The dynamic utility $c \mapsto U(c)$ is in certainty equivalent form relative to $\gamma$, or $\gamma$-CE for short, if at any time- $t$ information node, $U_{t}(c)$ represents the units of the cash flow $\gamma$ that result in the same time- $t$ utility as the consumption plan $c$. More formally, given any time $t$, let the set $\mathscr{C}_{t}^{\gamma}$ consist of all consumption plans $c$ such that $c_{s} / \gamma_{s}=$ $c_{t} / \gamma_{t}$ for all $s \geqslant t$. The dynamic utility $U$ is defined to be $\gamma$-CE if for any time $t$ and any $c \in \mathscr{C}_{t}^{\gamma}, U_{t}(c) \gamma_{t}=c_{t}$. To interpret this, consider any $c \in \mathscr{C}$. By our earlier assumption, there exists some $\bar{c} \in \mathscr{C}$ such that $\bar{c}_{s}=U_{t}(c) \gamma_{s}$ for all $s \geqslant t$, and therefore $\bar{c} \in \mathscr{C}_{t}^{\gamma}$. If $U$ is $\gamma$-CE, then $U_{t}(\bar{c}) \gamma_{t}=\bar{c}_{t}=U_{t}(c) \gamma_{t}$, and therefore $U_{t}(\bar{c})=U_{t}(c)$. In this sense, the cash flow $\gamma$ is the yardstick relative to which utility is cardinalized. 
Suppose now that $c \mapsto U(c)$ is both $\gamma$-CE and $\gamma$-TI. Then the quasi-linearity restriction (10) is satisfied. To see this, fix any $c \in \mathscr{C}$, any time $t$, and any scalar $k$. Select a $\bar{c} \in \mathscr{C}$ such that $\bar{c}_{s}=U_{t}(c) \gamma_{s}$ for $s \geqslant t$. Arguing as above, $\bar{c} \in \mathscr{C}_{t}^{\gamma}$ and $U_{t}(\bar{c})=$ $U_{t}(c)$. Since $U$ is $\gamma$-TI, it follows that $U_{t}(\bar{c}+k \gamma)=U_{t}(c+k \gamma)$. Since $U$ is $\gamma$-CE and $\bar{c}+k \gamma \in \mathscr{C}_{t}^{\gamma}, \quad U_{t}(\bar{c}+k \gamma) \gamma_{t}=\bar{c}_{t}+k \gamma_{t}$. Therefore, $U_{t}(c+k \gamma)=U_{t}(\bar{c}+k \gamma)=\bar{c}_{t} / \gamma_{t}+$ $k=U_{t}(c)+k$, proving Eq. (10).

Let $U^{k}=U(c+k \gamma)$ and $U=U^{0}=U(c)$. Since $U^{k}=U+k$, the volatility process of $U^{k}$ is $\Sigma^{U}$ for any scalar $k$. The definition of $U(c+k \gamma)$ implies

$$
\mathrm{d} U_{t}^{k}=-F\left(t, c_{t}+k \gamma_{t}, U_{t}^{k}, \Sigma_{t}^{U}\right) \mathrm{d} t+\Sigma_{t}^{U \prime} \mathrm{d} B_{t} .
$$

On the other hand, since $U^{k}=U+k$, the definition of $U(c)$ gives

$$
\mathrm{d} U_{t}^{k}=-F\left(t, c_{t}, U_{t}, \Sigma_{t}^{U}\right) \mathrm{d} t+\Sigma_{t}^{U \prime} \mathrm{d} B_{t},
$$

implying $F\left(t, c_{t}+k \gamma_{t}, U_{t}+k, \Sigma_{t}^{U}\right)=F\left(t, c_{t}, U_{t}, \Sigma_{t}^{U}\right)$. The last equality holds almost everywhere for any given value of $k$. Ignoring some uninteresting technicalities regarding null events, we formally set $k=-U_{t}$ and define the function $G(\omega, t, x, y, z)=F(\omega, t, \gamma(\omega, t) x, 0, z)$ to find

$$
F\left(t, c_{t}, U_{t}, \Sigma_{t}^{U}\right)=G\left(t, \frac{c_{t}}{\gamma_{t}}-U_{t}, \Sigma_{t}^{U}\right) .
$$

Noting that $U_{T}=c_{T} / \gamma_{T}$ ( $U$ being $\gamma$-CE), we obtain the BSDE of Condition 6:

$$
\mathrm{d} U_{t}=-G\left(t, \frac{c_{t}}{\gamma_{t}}-U_{t}, \Sigma_{t}^{U}\right) \mathrm{d} t+\Sigma_{t}^{U^{\prime}} \mathrm{d} B_{t}, \quad U_{T}=\frac{c_{T}}{\gamma_{T}} .
$$

The above conclusions are isomorphic to corresponding conclusions in SS with regard to homotheticity, via the transformations $\tilde{c}_{t}=\exp \left(-c_{t} / \gamma_{t}\right)$ and $\tilde{U}_{t}(\tilde{c})=$ $\exp \left(-U_{t}(c)\right)$, in which case $U$ is $\gamma$-TI if and only if $\tilde{U}$ is homothetic, $\tilde{U}$ is 1-CE if and only if $U$ is $\gamma$-CE, and $\tilde{U}$ is homogeneous of degree one if and only if $U$ satisfies the quasi-linearity property (10). The dynamics

$$
\mathrm{d} \tilde{U}_{t} / \tilde{U}_{t}=-\tilde{G}\left(t, \tilde{c}_{t} / \tilde{U}_{t}, \tilde{\sigma}_{t}\right) \mathrm{d} t+\tilde{\sigma}_{t}^{U \prime} \mathrm{d} B_{t}, \quad \tilde{U}_{t}=\tilde{c}_{T},
$$

in SS can be mapped to the above dynamics for $U$ by letting $G(\omega, t, x, y)=$ $-\tilde{G}(\omega, t, \exp (-x),-y)-y^{\prime} y / 2$, and applying Itô's lemma.

\section{Appendix B. Proofs}

\section{B.1. Proof of Theorem 3}

(a) Sufficiency: Given any feasible plan $(\tilde{c}, \tilde{\phi}, \tilde{W})$ and stopping time $\tau$, integration by parts, the dynamics of $\pi$, and the budget equation imply

$$
\begin{aligned}
\pi_{\tau} \tilde{W}_{\tau}-\pi_{0} w_{0}= & \int_{0}^{\tau} \pi_{t} \tilde{\phi}_{t}^{\prime} \varepsilon_{t} \mathrm{~d} t-\int_{0}^{\tau} \pi_{t}\left(\tilde{c}_{t}-e_{t}\right) \mathrm{d} t \\
& +\int_{0}^{\tau} \pi_{t}\left(\sigma_{t}^{R} \tilde{\phi}_{t}-\tilde{W}_{t} \eta_{t}\right)^{\prime} \mathrm{d} B_{t},
\end{aligned}
$$


and therefore

$$
\pi_{\tau} \tilde{W}_{\tau}-\pi_{0} w_{0} \leqslant \int_{0}^{\tau} \pi_{t} \delta_{\Phi}\left(\varepsilon_{t}\right) \mathrm{d} t-\int_{0}^{\tau} \pi_{t}\left(\tilde{c}_{t}-e_{t}\right) \mathrm{d} t+M_{t}^{1}
$$

for a local martingale $M^{1}$. Given condition (4), the same argument applied at $(c, \phi, W)$ gives

$$
\pi_{\tau} W_{\tau}-\pi_{0} w_{0}=\int_{0}^{\tau} \pi_{t} \delta_{\Phi}\left(\varepsilon_{t}\right) \mathrm{d} t-\int_{0}^{\tau} \pi_{t}\left(c_{t}-e_{t}\right) \mathrm{d} t+M_{t}^{2}
$$

for another local martingale $M^{2}$. Letting $x=\tilde{c}-c$, and $M=M^{1}-M^{2}$, we conclude that

$$
\pi_{\tau} \tilde{W}_{\tau}-\pi_{\tau} W_{\tau} \leqslant-\int_{0}^{\tau} \pi_{t} x_{t} \mathrm{~d} t+M_{\tau} .
$$

Let $\left\{\tau_{n}: n=1,2, \ldots\right\}$ be an increasing sequence of stopping times that converges to $T$ and such that $M$ stopped at $\tau_{n}$ is a martingale. Taking expectations in the last inequality, we find

$$
0 \geqslant E\left[\int_{0}^{\tau_{n}} \pi_{t} x_{t} \mathrm{~d} t+\pi_{\tau_{n}} \tilde{W}_{\tau_{n}}-\pi_{\tau_{n}} W_{\tau_{n}}\right] .
$$

The idea is to let $n \rightarrow \infty$ to conclude that $(\pi \mid x) \leqslant 0$. There remains to justify the interchange of limits and integration. For the integral of $\pi x$, we apply the dominated convergence theorem, using the assumptions $\pi, x \in \mathscr{H}$. For the term, involving $\pi \tilde{W}$, we use the assumptions $\pi \in \mathscr{S}_{2}$ and $\tilde{W}^{-} \in \mathscr{S}_{2}$, and Fatou's lemma to conclude that

$$
\lim \inf _{t \rightarrow \infty} E\left[\pi_{\tau_{n}} \tilde{W}_{\tau_{n}}\right] \geqslant E\left[\pi_{T} \tilde{W}_{T}\right] .
$$

Finally, the assumption $\pi W \in \mathscr{S}_{1}$ implies that $E\left[\pi_{\tau_{n}} W_{\tau_{n}}\right]$ converges to $E\left[\pi_{T} W_{T}\right]$. Taking the limit inferior on both sides of inequality (24), we therefore reach the conclusion $(\pi \mid x) \leqslant 0$.

(b) Necessity: By taking the accumulated value of a unit invested at time zero in the money market as the numeraire, we assume without loss in generality that $r=0$.

We begin by showing that $\pi$ is a martingale. Consider any time $t<T$ and any event $F \in \mathscr{F}_{t}$. Given any $h \in(t, T-t)$, consider the feasible plans $(c+x, \theta, W+z)$ and $(c-x, \theta, W-z)$, where $x$ is equal to the indicator function of $F \times[t, t+h]$, while $z$ is given by $\mathrm{d} z_{s}=-x_{s} \mathrm{~d} t, z_{0}=0$. Since $\pi$ is a state-price density, it follows that $(\pi \mid x)=$ 0 , and therefore

$$
E\left[1_{F}\left(\frac{1}{h} \int_{t}^{t+h} \pi_{s} \mathrm{~d} s-\pi_{T}\right)\right]=0 .
$$

Since $\pi$ is continuous and $\pi \in \mathscr{S}_{1}$, taking the limit implies that $E\left[1_{F} \pi_{t}\right]=E\left[1_{F} \pi_{T}\right]$. Applying this condition over all $t$ and $F \in \mathscr{F}_{t}$ implies that $\pi$ is a martingale. 
Since $\pi \in \mathscr{S}_{1}$ is a strictly positive Itô process and a martingale, there exists $\eta \in$ $\mathscr{L}_{2}\left(\mathbb{R}^{d}\right)$ such that

$$
\frac{\mathrm{d} \pi_{t}}{\pi_{t}}=-\eta_{t}^{\prime} \mathrm{d} B_{t}
$$

Defining $\varepsilon_{t}=\mu_{t}^{R}-\sigma_{t}^{R \prime} \eta_{t}$, we complete the proof by showing that $\phi_{t}^{\prime} \varepsilon_{t}=\delta_{\Phi}\left(\varepsilon_{t}\right)$. We first consider a lemma suggestive of the result:

Lemma A.1. Suppose that $(c, \phi, W)$ and $(c+x, \phi+y, W+z)$ are feasible plans, $y_{T}=$ $0, y^{\prime} \varepsilon \in \mathscr{H}$ and $\pi z \in \mathscr{S}_{1}$. Then $(\pi \mid x)=\left(\pi \mid y^{\prime} \varepsilon\right)$.

Proof. As in the sufficiency proof, for any feasible plan $(\tilde{c}, \tilde{\phi}, \tilde{W})$ and stopping time $\tau$, Eq. (23) holds. Taking the difference of the equation applied at $(c+x, \phi+y, W+$ $z)$, and the equation applied at $(c, \phi, W)$, we find that

$$
\int_{0}^{\tau} \pi_{t} x_{t} \mathrm{~d} t+\pi_{\tau} z_{\tau}=\int_{0}^{\tau} \pi_{t} y_{t}^{\prime} \varepsilon_{t} \mathrm{~d} t+M_{\tau}
$$

for some local martingale $M$. Consider now an increasing sequence of stopping times $\left\{\tau_{n}: n=1,2, \ldots\right\}$ that converges to $T$, such that $M$ stopped at $\tau_{n}$ is a martingale. It then follows that

$$
E\left[\int_{0}^{\tau_{n}} \pi_{t} x_{t} \mathrm{~d} t+\pi_{\tau_{n}} z_{\tau_{n}}\right]=E\left[\int_{0}^{\tau_{n}} \pi_{t} y_{t}^{\prime} \varepsilon_{t} \mathrm{~d} t\right], \quad n=1,2 \ldots .
$$

Feasibility of $(c+x, \phi+y, W+z)$ implies that $x_{T}=z_{T}$, and therefore the left-hand side converges to $(\pi \mid x)$, given the assumptions that $\pi z \in \mathscr{S}_{1}$ and $\pi, x \in \mathscr{H}$. The righthand side converges to $\left(\pi \mid y^{\prime} \varepsilon\right)$ since $\pi, y^{\prime} \varepsilon \in \mathscr{H}$.

Finally, we show that, for every process $\phi+y$ valued in $\Phi, y_{t}^{\prime} \varepsilon_{t} \leqslant 0$. Suppose instead that there exists some process $y$ such that $\tilde{\phi}=\phi+y$ is valued in $\Phi$ but $E\left[\int_{0}^{T} 1\left\{y_{t}^{\prime} \varepsilon_{t}>0\right\} \mathrm{d} t\right]>0$. For each positive integer $N$, define

$$
y_{t}^{N}=y_{t} 1\left\{0 \leqslant y_{t}^{\prime} \varepsilon_{t} \leqslant N,\left\|y_{t}\right\|<N\right\} .
$$

Since $1\left\{y_{t}^{N \prime} \varepsilon_{t}>0\right\}$ monotonically converges almost surely to $1\left\{y_{t}^{\prime} \varepsilon_{t}>0\right\}$, it follows that $E\left[\int_{0}^{T} 1\left\{y_{t}^{N \prime} \varepsilon_{t}>0\right\} \mathrm{d} t\right]>0$ for some $N$. We therefore assume, without loss in generality, that $y^{\prime} \varepsilon$ is nonnegative and bounded, and $y$ is bounded, which in turn implies that $\tilde{\phi}$ is a trading plan. Similarly, for every positive integer $N$, define the stopping time

$$
\tau^{N}=\inf \left\{t: \int_{0}^{t} y_{s}^{\prime} \mathrm{d} R_{s}>N \text { or } T=T\right\}
$$

and the process $y_{t}^{N}=y_{t} 1\left\{t<\tau_{N}\right\}$. The same convergence argument used above shows that $E\left[\int_{0}^{T} 1\left\{y_{t}^{N \prime} \varepsilon_{t}>0\right\} \mathrm{d} t\right]>0$ for some $N$. We therefore also assume, without loss in generality, that the process $z$, defined by $d z_{t}=y_{t}^{\prime} \mathrm{d} R_{t}, z_{0}=0$, is bounded, and therefore $W+z$ is a wealth process. Letting $x_{t}=0$ for $t<T$, and $x_{T}=z_{T}$, it follows that $(c+x, \phi+y, W+z)$ is a feasible plan. Applying Lemma A.1, it follows that $(\pi \mid x)=\left(\pi \mid y^{\prime} \varepsilon\right)>0$, implying that $\pi$ cannot be a state-price density. 


\section{B.2. Proof of Theorem 9}

(a) Sufficiency: We begin by confirming the budget equation and that $U=U(c)$. Given the construction of $U$ and $\phi$ in (12), and Eq. (13), the wealth dynamics (14) are easily confirmed to be equivalent to the budget equation (1). If $\mathrm{d} U_{t}=\ldots \mathrm{d} t+$ $\Sigma_{t}^{U \prime} \mathrm{d} B_{t}$, integration by parts applied to $Y=U \Gamma-W$ implies

$$
\Sigma_{t}^{U}=\frac{\Sigma_{t}^{Y}+\sigma_{t}^{R} \phi_{t}^{0}}{\Gamma_{t}} \quad \text { and } \quad x_{t}=\frac{c_{t}}{\gamma_{t}}-U_{t}=\mathscr{X}\left(t, \frac{\gamma_{t}}{\Gamma_{t}}, \Sigma_{t}^{U}\right) .
$$

To verify that $U=U(c)$, we use the above expression for $\Sigma^{U}$, and the fact that

$$
G^{*}\left(t, \frac{\gamma_{t}}{\Gamma_{t}}, \Sigma\right)=G\left(t, x_{t}, \Sigma\right)-\frac{\gamma_{t}}{\Gamma_{t}} x_{t},
$$

to rewrite the dynamics of $Y$ as

$$
\mathrm{d} Y_{t}=\left(Y_{t} r_{t}-\phi_{t}^{0 \prime} \mu_{t}^{R}-e_{t}-\Gamma_{t} G\left(t, x_{t}, \Sigma_{t}^{U}\right)+\gamma_{t} x_{t}+\left(\sigma_{t}^{R} \varrho\right)^{\prime} \Sigma_{t}^{U}\right) \mathrm{d} t+\Sigma_{t}^{Y^{\prime}} \mathrm{d} B_{t} .
$$

Integration by parts applied to $Y=U \Gamma-W$, simplified using the dynamics of $Y, \Gamma$, and $W$, and identities (12) and (13), results in the utility dynamics (11), confirming that $U=U(c)$.

Recalling that $\phi=\phi^{0}+U \varrho \in \Phi$ if and only if $\phi^{0} \in \Phi$, we therefore have a feasible plan $(c, \phi, W)$, with $U=U(c)$. We prove optimality of this plan by applying Proposition 2, showing that the process $\pi=\mathscr{E} / \Gamma$ is both a supergradient density of $U_{0}$ at $c$ and a state-price density at $c$.

Let $\lambda_{t}=F_{c}\left(t, c_{t}, U_{t}, \Sigma_{t}^{U}\right)$. By Proposition 13 of Schroder and Skiadas [32], we know that if $\left(F_{U}, F_{\Sigma}\right) \in \mathscr{L}_{1}(\mathbb{R}) \times \mathscr{L}_{2}\left(\mathbb{R}^{d}\right)$ is such that $\mathscr{E}\left(F_{U}, F_{\Sigma}\right) \in \mathscr{S}_{2}$ and $\left(F_{U}(t), F_{\Sigma}(t)\right) \in\left(\partial_{U, \Sigma} F\right)\left(t, c_{t}, U_{t}, \Sigma_{t}^{U}\right)$ for all $t$, and $\pi=\mathscr{E}\left(F_{U}, F_{\Sigma}\right) \lambda$ defines an element of $\mathscr{H}$, then $\pi$ is a supergradient density of $U_{0}$ at $c$. The aggregator form implies $\lambda_{t}=\gamma_{t}^{-1} G_{x}\left(t, x_{t}, \Sigma_{t}^{U}\right)$, and therefore, by $(25), \lambda=1 / \Gamma$. The aggregator form also implies that $\left(F_{U}(t), F_{\Sigma}(t)\right) \in\left(\partial_{U, \Sigma} F\right)\left(t, c_{t}, U_{t}, \Sigma_{t}^{U}\right)$ if and only if $F_{U}(t)=-G_{x}\left(t, x_{t}, \Sigma_{t}^{U}\right)\left(=-\gamma_{t} / \Gamma_{t}\right)$ and $F_{\Sigma}(t) \in \partial_{\Sigma} G\left(t, x_{t}, \Sigma_{t}^{U}\right)$. Putting the above observations together confirms that $\pi$ is a supergradient density of $U_{0}$ at $c$.

Finally, we use part (a) of Theorem 3 to confirm that $\pi$ is a state-price density at $c$. If $\mathrm{d} \lambda_{t} / \lambda_{t}=\mu_{t}^{\lambda} \mathrm{d} t+\sigma_{t}^{\lambda \prime} \mathrm{d} B_{t}$, then, using the fact that $\lambda=1 / \Gamma$,

$$
\mu_{t}^{\lambda}=-r_{t}+\frac{\gamma_{t}}{\Gamma_{t}}-\frac{\varrho^{\prime} \mu_{t}^{R}}{\Gamma_{t}}+\sigma_{t}^{\lambda \prime} \sigma_{t}^{\lambda} \quad \text { and } \quad \sigma_{t}^{\lambda}=-\frac{\sigma_{t}^{R} \varrho}{\Gamma_{t}} .
$$

Since trading in $\varrho$ is unrestricted, $\delta_{\Phi}(\varepsilon)<\infty$ implies $\varrho^{\prime} \varepsilon=0$, and therefore $\varrho^{\prime} \mu^{R} / \Gamma$ $\sigma^{\lambda \prime} \sigma^{\lambda}=\sigma^{\lambda \prime} G_{\Sigma}$, resulting in $\mu^{\lambda}=-r+\gamma / \Gamma-\sigma^{\lambda \prime} G_{\Sigma}$. Using this fact and applying integration by parts to $\pi=\mathscr{E} \lambda$, we obtain condition (4), where $\eta=-\left(G_{\Sigma}-\Gamma^{-1} \sigma^{R} \varrho\right)$. We have therefore verified that $\pi$ is a state-price density at $c$, completing the optimality verification.

(b) Necessity: We begin by proving that $\lambda=1 / \Gamma$. Since $G$ is smooth, by Proposition 13 of Schroder and Skiadas [32], $\pi \in \mathscr{E} \lambda$, where $\lambda_{t}=G_{x}\left(t, x_{t}, \Sigma_{t}\right) / \gamma_{t}$, defines a utility gradient density. By Proposition $2, \pi$ is also a state-price density at $c$. 
Since trading the portfolio $\varrho$ is unrestricted,

$$
\Gamma_{t}=\frac{1}{\pi_{t}} E_{t}\left[\int_{t}^{T} \pi_{s} \gamma_{s} \mathrm{~d} s+\pi_{T} \gamma_{T}\right] .
$$

This follows by a standard argument that we now outline. Suppose, without loss of generality (by taking one unit invested initially in the money market as the numeraire), that $r=0$. We have shown in the proof of part (b) of Theorem 3 that $\pi$ is a martingale. Suppose now that at time $t$ and event $F \in \widetilde{F}_{t}$ the agent borrows from the money market to buy portfolio $\varrho$, consuming the ensuing dividend stream $\gamma$, and paying back the loan $\Gamma_{t}$ by reducing terminal consumption. The resulting feasible incremental cash flow $x$ is given by $x_{s}=0$ for $s<t, x_{s}=1_{F} \gamma_{s}$ for $s \in(t, T)$, and $x_{T}=1_{F}\left(\gamma_{T}-\Gamma_{t}\right)$. Since $-x$ is also feasible, we have

$$
0=(\pi \mid x)=E\left[1_{F}\left(\int_{t}^{T} \pi_{s} \gamma_{s} \mathrm{~d} s+\pi_{T}\left(\gamma_{T}-\Gamma_{t}\right)\right)\right] .
$$

Since $F \in \mathscr{F}_{t}$ is arbitrary, it follows that $E_{t}\left[\int_{t}^{T} \pi_{s} \gamma_{s} \mathrm{~d} s+\pi_{T} \gamma_{T}\right]=\Gamma_{t} E_{t}\left[\pi_{T}\right]=\Gamma_{t} \pi_{t}$, as claimed.

Taking the derivative of the function $f(k)=U(c+k \gamma)$ at zero, we obtain

$$
\mathscr{E}_{t} f_{t}^{\prime}(0)=E_{t}\left[\int_{t}^{T} \pi_{s} \gamma_{s} \mathrm{~d} s+\pi_{s} \gamma_{T}\right]=\pi_{t} \Gamma_{t} .
$$

Since $U(c+k \gamma)=k+U(c)$, it also the case that $f^{\prime}(0)=1$, and therefore $\Gamma=\mathscr{E} / \pi=$ $1 / \lambda$.

Having shown the key fact that $\lambda=1 / \Gamma$, we define the process $Y=U \Gamma-W$, and apply Itô's lemma, using the dynamics of $U$ and $\Gamma$, the definition of $G^{*}$ and $\mathscr{X}$, as well as the budget equation, to obtain

$$
\begin{aligned}
\mathrm{d} Y_{t}= & U_{t} \mathrm{~d} \Gamma_{t}+\Gamma_{t} \mathrm{~d} U_{t}+\Sigma^{U^{\prime}}\left(\sigma^{R} \varrho\right) \mathrm{d} t-\mathrm{d} W_{t} \\
= & \left(Y_{t} r_{t}-\phi_{t}^{0} \mu_{t}^{R}+\Sigma_{t}^{U \prime} \sigma_{t}^{R} \varrho-\Gamma_{t} G^{*}\left(t, \frac{\gamma_{t}}{\Gamma_{t}}, \Sigma_{t}^{U}\right)-e_{t}\right) \mathrm{d} t \\
& +\left(\Gamma_{t} \Sigma_{t}^{U}-\sigma^{R} \phi_{t}^{0}\right)^{\prime} \mathrm{d} B_{t} .
\end{aligned}
$$

The diffusion term confirms the expression for $\Sigma^{U}$ in (25), which when substituted into the drift term results in the dynamics of $Y$ in Condition 8 . The rest of the condition follows easily from the necessary conditions of Theorem 3 for $\pi$ to be a state-price density at $c$.

\section{B.3. Proof of Proposition 13}

Substituting $G_{\Sigma}=-\left(q+\kappa \otimes \Lambda+Q \Sigma^{U}\right), \Lambda \in \partial\left|\Sigma^{U}\right|$, into the equation for $\varepsilon$ in Condition 8 gives

$$
\varepsilon=\mu^{R}-\sigma^{R^{\prime}}\left(q+\kappa \otimes \Lambda+\frac{\sigma^{R} \varrho}{\Gamma}+Q \Sigma^{U}\right) .
$$


The formula for $\phi^{0}$ is obtained after substituting $\Sigma^{U}=\Gamma^{-1}\left(\Sigma^{Y}+\sigma^{R} \phi^{0}\right)$. Computing the inner product of $\varepsilon$ and $\phi^{0}$ results in

$$
\phi^{0 \prime} \mu^{R}=\delta_{\Phi}(\varepsilon)+\phi^{0 \prime} \sigma^{R \prime}\left(q+\kappa \otimes \Lambda+\Gamma^{-1} \sigma^{R} \varrho+Q \Sigma^{U}\right) .
$$

Letting $\mathrm{d} Y=\mu^{Y} \mathrm{~d} t+\Sigma^{Y \prime} \mathrm{d} B$, we substitute for $G^{*}$ in Condition 8 to find

$$
\mu^{Y}=Y r-\phi^{0 \prime} \mu^{R}-e-\Gamma g^{*}+\left(\sigma^{R} \varrho\right)^{\prime} \Sigma^{U}+\Gamma\left(q^{\prime} \Sigma^{U}+\kappa^{\prime}\left|\Sigma^{U}\right|+\frac{1}{2} \Sigma^{U \prime} Q \Sigma^{U}\right) .
$$

Using the fact that $\kappa^{\prime}\left|\Sigma^{U}\right|=\Sigma^{U^{\prime}}(\kappa \otimes \Lambda)$ and the above expression for $\phi^{0 \prime} \mu^{R}$, the drift term of $Y$ becomes $\mu^{Y}=Y r-e-\Gamma g^{*}-\delta_{\Phi}(\varepsilon)+\mathscr{Q}$, where

$$
\begin{aligned}
\mathscr{Q} & =\left(\Gamma \Sigma^{U^{\prime}}-\phi^{0 \prime} \sigma^{R \prime}\right)\left(q+\kappa \otimes \Lambda+\Gamma^{-1} \sigma^{R} \varrho\right)+\frac{1}{2} \Gamma \Sigma^{U \prime} Q \Sigma^{U}-\phi^{0 \prime} \sigma^{R \prime} Q \Sigma^{U} \\
& =\Sigma^{Y^{\prime}}\left(q+\kappa \otimes \Lambda+\Gamma^{-1} \sigma^{R} \varrho\right)+\frac{1}{2} \Gamma^{-1}\left(\Sigma^{Y \prime} Q \Sigma^{Y}-\phi^{0 \prime} \sigma^{R \prime} Q \sigma^{R} \phi^{0}\right) .
\end{aligned}
$$

\section{B.4. Proof of Proposition 21}

The expression for $\phi^{0}$ in (18) implies

$$
\Gamma^{-1} Q_{i i}\left(\Sigma^{Y}+\sigma^{R} \phi^{0}\right)_{i}=\alpha_{i}-\kappa_{i} \Lambda_{i}, \quad i \in M .
$$

Since $\Lambda_{i} \in \partial\left|\Sigma^{Y}+\sigma^{R} \phi^{0}\right|$, there are three possibilities: (1) $\left(\Sigma^{Y}+\sigma^{R} \phi^{0}\right)_{i}>0, \Lambda_{i}=1$, $\alpha_{i}>\kappa_{i}$; (2) $\left(\Sigma^{Y}+\sigma^{R} \phi^{0}\right)_{i}=0, \alpha_{i}=\kappa_{i} \Lambda_{i}$; and (3) $\left(\Sigma^{Y}+\sigma^{R} \phi^{0}\right)_{i}<0, \Lambda_{i}=-1, \alpha_{i}<-\kappa_{i}$. In all three cases, $\kappa_{M} \otimes \Lambda_{M}=\max \left\{\min \left\{\alpha_{M}, \kappa_{M}\right\},-\kappa_{M}\right\}$. Since $\left(\Sigma^{Y}+\sigma^{R} \phi^{0}\right)_{N}=\Sigma_{N}^{Y}$, we have $\Lambda_{N} \in \partial\left|\Sigma_{N}^{Y}\right|$ and therefore $\Sigma_{N}^{Y \prime}\left(\kappa_{N} \otimes \Lambda_{N}\right)=\Sigma_{N}^{Y \prime}\left(\kappa_{N} \otimes \operatorname{sign}\left(\Sigma_{N}^{Y}\right)\right)$. Finally, from $\Gamma U=Y+W$, we get $\Gamma \Sigma_{M}^{U}=\Sigma_{M}^{Y}+\sigma_{M M}^{R} \phi^{0}=\Gamma\left(Q_{M M}\right)^{-1} \hat{\alpha}_{M}$.

\section{B.5. Proof of Proposition 23}

Substituting the expression for $\phi^{0}$ in (18) into the equation for $Y$ in Proposition 13, we find

$$
\begin{aligned}
\mathrm{d} Y= & \left(Y r-e-\Gamma g^{*}\left(\frac{\gamma}{\Gamma}\right)-\frac{\Gamma}{2} \hat{\alpha}^{\prime} Q_{M M}^{-1} \hat{\alpha}+\frac{1}{2 \Gamma} \Sigma_{N}^{Y^{\prime}} Q_{N N} \Sigma_{N}^{Y}\right) \mathrm{d} t \\
& +\Sigma^{Y^{\prime}}\left[\mathrm{d} B+\left(\begin{array}{c}
\eta_{M} \\
q_{N}+\kappa_{N} \otimes \Lambda_{N}
\end{array}\right) \mathrm{d} t\right] .
\end{aligned}
$$

Suppose $Y_{t}^{e}$ satisfies the stated BSDE and $\Sigma_{N}^{M}=0$. Then direct computation shows that $Y_{t}^{M}=Y-Y_{t}^{e}$ satisfies the claimed BSDE.

\section{B.6. Proof of Example 25}

Only the proof Case 2 is presented. The proof of Case 1 is similar. The assumptions of the example imply that $e$ is the only source of uncertainty driving $Y_{t}^{e}$, and therefore $\Sigma_{t}^{Y^{e}}=v_{t} \Sigma$ for some scalar-valued process $v$. Therefore,

$$
\mathrm{d} Y_{t}^{e}=-\left(e_{t}-Y_{t}^{e} r_{t}-\frac{a}{2 \Gamma_{t}} v_{t}^{2} \Sigma^{\prime} \Sigma\right) \mathrm{d} t+v_{t} \Sigma^{\prime} \mathrm{d} B_{t}^{\theta} .
$$


Defining $z_{t}=\exp \left(-a Y_{t}^{e} / \Gamma_{t}\right)$, we have

$$
\begin{aligned}
\frac{\mathrm{d} z_{t}}{z_{t}} & =-\frac{a}{\Gamma_{t}} \mathrm{~d} Y_{t}^{e}+\frac{1}{2}\left(\frac{a}{\Gamma_{t}}\right)^{2} v_{t}^{2} \Sigma^{\prime} \Sigma \mathrm{d} t-Y_{t}^{e} \mathrm{~d}\left(\frac{a}{\Gamma_{t}}\right) \\
& =\frac{a}{\Gamma_{t}}\left(e_{t}-Y_{t}^{e} r_{t}\right) \mathrm{d} t-\frac{a}{\Gamma_{t}} v_{t} \Sigma^{\prime} \mathrm{d} B_{t}^{\theta}-Y_{t}^{e} \mathrm{~d}\left(\frac{a}{\Gamma_{t}}\right) \\
& =-a Y_{t}^{e}\left(\mathrm{~d}\left(\frac{1}{\Gamma_{t}}\right)+\frac{r_{t}}{\Gamma_{t}} \mathrm{~d} t\right)+\frac{a}{\Gamma_{t}} e_{t} \mathrm{~d} t+\sigma_{t}^{z \prime} \mathrm{d} B_{t}^{\theta},
\end{aligned}
$$

where $\sigma_{t}^{z}=-\alpha v_{t} \Sigma^{e} / \Gamma_{t}$. Since $r$ and $\gamma$ are deterministic,

$$
\mathrm{d}\left(\frac{1}{\Gamma_{t}}\right)=-\left(\frac{r_{t}-\gamma_{t} / \Gamma_{t}}{\Gamma_{t}}\right) \mathrm{d} t
$$

and therefore $z$ solves the BSDE:

$$
\frac{\mathrm{d} z_{t}}{z_{t}}=\frac{1}{\Gamma_{t}}\left(a e_{t}-\gamma_{t} \log \left(z_{t}\right)\right) \mathrm{d} t+\sigma_{t}^{z \prime} \mathrm{d} B_{t}^{\theta}, \quad z_{T}=\exp \left(-\frac{a}{\Gamma_{T}} e_{T}\right) .
$$

Under the assumption that $\gamma_{t}=0, t<T$, the equation for $z$ in (20) is easily confirmed.

\section{B.7. Proof of Proposition 16 and Example 17}

We derive the consumption dynamics under the more general absolute aggregator form

$$
G(\omega, t, x, \Sigma)=g(\omega, t, x)+H(\omega, t, \Sigma),
$$

where $g_{x}(t, x)$ is assumed state independent. Letting $x_{t}=c_{t} / \gamma_{t}-U_{t}$, and applying Itô's lemma to $g_{x}\left(t, x_{t}\right)=\gamma_{t} / \Gamma_{t}$, gives

$$
g_{x t}\left(t, x_{t}\right) \mathrm{d} t+g_{x x}\left(t, x_{t}\right) \mathrm{d} x_{t}+\frac{1}{2} g_{x x x}\left(t, x_{t}\right)\left(\mathrm{d} x_{t}\right)^{2}=\mathrm{d}\left(\frac{\gamma_{t}}{\Gamma_{t}}\right) .
$$

Squaring this expression results in

$$
\left(\mathrm{d} x_{t}\right)^{2}=\left(\frac{\mathrm{d}\left(\gamma_{t} / \Gamma_{t}\right)}{g_{x x}\left(t, x_{t}\right)}\right)^{2}=\frac{1}{\mathscr{A}_{t}^{2}} \sigma_{t}^{\gamma / \Gamma \prime} \sigma_{t}^{\gamma / \Gamma} .
$$

Matching the diffusion terms in (26) and rearranging, we find that

$$
\frac{1}{\gamma_{t}} \Sigma_{t}^{c}=\frac{1}{\mathscr{A}_{t}} \frac{\sigma_{t}^{R} \varrho}{\Gamma_{t}}+\left(\frac{c_{t}}{\gamma_{t}}-\frac{1}{\mathscr{A}_{t}}\right) \sigma_{t}^{\gamma}+\Sigma_{t}^{U}
$$

Substituting $\Sigma^{U}=\Gamma^{-1}\left(\Sigma^{Y}+\sigma^{R} \phi-\sigma^{R} \varrho U\right)$, and rearranging gives the formula for $\Sigma^{c}$.

The formula for $\mu_{t}^{c}$ is obtained similarly by matching the drift terms in Eq. (26), and the equilibrium short rate is obtained by inverting and solving for $r$ as a function of $\mu_{t}^{c}$. The risk premium expression is derived by solving (27) for $\Sigma^{U}$, and 
substituting into

$$
\mu_{t}^{R}=-\sigma_{t}^{R^{\prime}}\left(H_{\Sigma}\left(t, \Sigma^{U}\right)-\frac{\sigma_{t}^{R} \varrho}{\Gamma_{t}}\right),
$$

which follows from the first-order conditions with $\varepsilon=0$. The quasi-quadratic case corresponds to $H_{\Sigma}\left(t, \Sigma^{U}\right)=-Q_{t} \Sigma^{U}$.

\section{References}

[1] G. Chacko, L. Viceira, Dynamic consumption and portfolio choice with stochastic volatility in incomplete markets, Working Paper, Harvard University, 1999.

[2] Z. Chen, L. Epstein, Ambiguity, risk, and asset returns in continuous time, Econometrica 70 (2002) $1403-1443$.

[3] J. Cox, C.-F. Huang, Optimal consumption and portfolio policies when asset prices follow a diffusion process, J. Econ. Theory 49 (1989) 33-83.

[4] D. Cuoco, Optimal consumption and equilibrium prices with portfolio constraints and stochastic income, J. Econ. Theory 72 (1997) 33-73.

[5] J. Cvitanić, I. Karatzas, Convex duality in constrained portfolio optimization, Ann. Appl. Probab. 2 (1992) 767-818.

[6] D. Duffie, L. Epstein, Stochastic differential utility, Econometrica 60 (1992) 353-394.

[7] D. Duffie, D. Filipovic, W. Schachermayer, Affine processes and applications in finance, Ann. Appl. Probab. 13 (2003) 984-1053.

[8] D. Duffie, V. Fleming, M. Soner, T. Zariphopoulou, Hedging in incomplete markets with HARA utility, J. Econ. Dynam. Control 21 (1997) 753-782.

[9] D. Duffie, C. Skiadas, Continuous-time security pricing: a utility gradient approach, J. Math. Econ. 23 (1994) 107-131.

[10] N. El Karoui, S. Peng, M.-C. Quenez, A dynamic maximum principle for the optimization of recursive utilities under constraints, Ann. Appl. Probab. 2001, 664-693.

[11] D. Ellsberg, Risk, ambiguity, and the savage axioms, Q. J. Econ. 75 (1961) 643-669.

[12] L. Epstein, Behavior under risk: recent developments in theory and applications, in: J.-J. Laffont (Ed.), Advances in Economic Theory, Cambridge University Press, Cambridge, UK, 1992.

[13] L. Epstein, J. Miao, A two-person dynamic equilibrium under ambiguity, Working Paper, Department of Economics, University of Rochester, Rochester, NY, 2000.

[14] H. He, N. Pearson, Consumption and portfolio policies with incomplete markets and short-sale constrains: the infinite dimensional case, J. Econ. Theory 54 (1991) 259-304.

[15] S. Heston, A closed-form solution for options with stochastic volatility with applications to bond and currency options, Rev. Financial Stud. 6 (1993) 327-344.

[16] J. Hugonnier, D. Kramkov, Optimal investment with random endowments in incomplete markets, Working Paper, HEC Montreal and Carnegie Mellon University, 2002.

[17] I. Karatzas, J. Lehoczky, S. Shreve, Optimal portfolio and consumption decisions for a 'small investor' on a finite horizon, SIAM J. Control Optim. 25 (1987) 1557-1586.

[18] I. Karatzas, J. Lehoczky, S. Shreve, G. Xu, Martingale and duality methods for utility maximization in an incomplete market, SIAM J. Control Optim. 29 (1991) 702-730.

[19] T. Kim, E. Omberg, Dynamic nonmyopic portfolio behavior, Rev. Financial Stud. 9 (1996) 141-162.

[20] P. Klibanoff, M. Marinacci, S. Mukerji, A smooth model of decision making under ambiguity, Working Paper, MEDS, Kellogg School of Management, Northwestern University, 2002.

[21] H. Koo, Nontraded assets in incomplete markets, Math. Finance 8 (1998) 49-65.

[22] D. Kramkov, W. Schachermayer, The asymptotic elasticity of utility functions and optimal investment in incomplete markets, Ann. Appl. Probab. 9 (1999) 904-950.

[23] A. Lazrak, M.C. Quenez, A generalized stochastic differential utility, Math. Oper. Res. 28 (2003) $154-180$. 
[24] J. Liu, Portfolio selection in stochastic environments, Working Paper, UCLA, 2001.

[25] J. Ma, P. Protter, J. Yong, Solving forward-backward stochastic differential explicitly - a four step scheme, Probab. Theory Related Fields 98 (1994) 339-359.

[26] J. Ma, J. Yong, Forward-Backward Stochastic Differential Equations and Their Applications, Springer, Berlin, 1999.

[27] R. Merton, Optimum consumption and portfolio rules in a continuous-time model, J. Econ. Theory 3 (1971) 373-413.

[28] M. Musiela, T. Zariphopoulou, An example of indifference prices under exponential preferences, Finance Stochastics 8 (2004) 229-239.

[29] M. Piazzes, Affine term structure models, in: Handbook of Financial Econometrics, Elsevier, NorthHolland, Amsterdam, in press.

[30] M. Schroder, C. Skiadas, Optimal consumption and portfolio selection with stochastic differential utility, J. Econ. Theory 89 (1999) 68-126.

[31] M. Schroder, C. Skiadas, An isomorphism between asset pricing models with and without linear habit formation, Rev. Financial Stud. 15 (2002) 1189-1221.

[32] M. Schroder, C. Skiadas, Optimal lifetime consumption-portfolio strategies under trading constraints and generalized recursive preferences, Stochastic Process. Appl. 108 (2003) 155-202.

[33] M. Schroder, C. Skiadas, Quasiarbitrage, optimality, state pricing and duality in constrained financial markets with continuous and discontinuous information, Working Paper 327, Department of Finance, Kellogg School of Management, Northwestern University, 2004.

[34] U. Segal, A. Spivak, First-order versus second-order risk aversion, J. Econ. Theory 51 (1990) $111-125$.

[35] S. Shreve, G. Xu, A duality method for optimal consumption and investment under short-selling prohibition: I, general market coefficients; and II, constant market coefficients, Ann. Appl. Probab. 2 (1992) 87-112 and 314-328.

[36] C. Skiadas, Advances in the theory of choice and asset pricing, Ph.D. Thesis, Stanford University, 1992.

[37] C. Skiadas, Robust control and recursive utility, Finance Stochastics 7 (2003) 475-489.

[38] L. Svensson, I. Werner, Nontraded assets in incomplete markets: pricing and portfolio choice, European Econ. Rev. 37 (1993) 1149-1168.

[39] J. Yong, X.Y. Zhou, Stochastic Controls: Hamiltonian Systems and HJB Equations, Springer, New York, 1999. 